McGill/93-20

hep-ph/9308289

(June, 1993)

\title{
SPINOR HELICITY TECHNIQUE AND STRING REORGANIZATION FOR MULTILOOP DIAGRAMS ${ }^{\dagger}$
}

\author{
C.S. Lam* \\ Department of Physics, McGill University, 3600 University St. \\ Montreal, P.Q., Canada H3A 2T8
}

\begin{abstract}
Methods to apply the spinor helicity technique and string reorganization to multiloop amplitudes using the Feynman- and Schwinger-parameter representations are reviewed and expanded.
\end{abstract}

* Email address: Lam@physics.mcgill.ca

$\dagger$ To be published in the Canadian Journal of Physics 


\section{Introduction}

Perturbation theory is taught in every text book but its actual calculation could be very complicated. This is particularly so for gauge theories where the presence of spin, and possibly color, come in to tangle up the algebra. The fact that many gauge-dependent terms occur in the intermediate steps do not help the situation either. Such complexity grows at an alarming rate with the number $n$ of external particles and with the number $\ell$ of loops. For example, the number of terms in a QCD $2 \rightarrow 6$ gluon process is estimated already to be half a billion, even when no loop nor fermion is present. This is a serious problem that cannot be ignored because large $n$ and $\ell \neq 0$ calculations are unavoidable in high energy and high precision experiments. If polarized cross-sections are measured helicity amplitudes are needed as well, and this adds further to the complications. With such an amount of complexity even large computers are powerless. This makes it imperative to find new ways to overcome or to reduce computational difficulties in loops, with large $n$, and for polarized amplitudes.

In this connection there were two important advances in the last decade. This is the discovery of the spinor helicity technique [1-21] and the realization of the power of superstring calculations [22-25]. With the help of these new techniques previously uncalculable processes can now be computed. For example, exact tree amplitudes for $e^{+}+e^{-} \rightarrow \mu^{+}+\mu^{-}+n \gamma$ can now be obtained provided all photons carry the same helicity. Exact $n$-gluon tree amplitudes in the absence of fermions are given by the Parke-Taylor formula, which is valid if all but at most two of the $n$ outgoing gluons have the same helicities. These and other tree processes were calculated using the spinor helicity technique [1-21]. One-loop four and five polarized gluon amplitudes have now been obtained from superstring calculations [22-25].

In its original form, the spinor helicity technique cannot be used beyond the tree approximation. It is also difficult to carry out superstring calculations beyond one loop or to include external fermions. These limitations have now been overcome using parametric representations $[26,27]$. As a result, spinor helicity technique can now be applied to any number of loops [26]. A field-theoretic substitute for superstring calculations is now available to allow simplifications found in one-loop superstring calculations to be extended to multiloop amplitudes, with or without external fermions [27]. We shall call this field-theoretic substitute string reorganization.

The main purpose of this paper is to review these two recent developments. Although most of the crucial points have been made before $[26,27]$, there are nevertheless a number of new formulas, new proofs, and new details added to this article. This includes the use of spinor paths in Sec. 4, the new differential circuit identities in Appendix A and the corresponding new proof of the stringlike formulas. A technical restriction appeared earlier [27] governing the use of the multiloop stringlike formula has now been removed, and stringlike formulas have also been generalized to QCD. Local gauge invariance for internal vertices are shown in Appendix D, a new interpretation of gauge invariance as proper-time reparametrization invariance has been put forward. Finally, a perturbative proof of the off-shell Gordon identity is given in Appendix E. 
In order to fulfil the dual role of a review article, that of giving an elementary introduction to the basic ideas and results, but at the same time being reasonably detailed and self-contained to be useful to workers in the field, the article is divided into the main text and the appendices. The main text, from Sec. 2 to Sec. 5, contains a discussion of the motivations, the problems, and the solutions. The proofs and other finer details are to be found in the appendices.

The methods to be discussed in this article are applicable to any gauge theory, but for simplicity of presentation we shall confine ourselves here to QED and QCD. In the rest of this section, we shall give a brief account of these two new developments.

The spinor helicity technique makes use of the fact that external fermion masses can be neglected in high energy collisions. In that case helicity and chirality are both conserved and the multichannel spin problem reduces to a one-channel problem (see Sec. 4.1 for details). The technique can be used as long as all the internal momenta are linear combinations of the massless external momenta, which is so for tree but not for loop amplitudes. This is why in its original form the technique can only be applied to tree but not loop diagrams. There is however nothing against internal particles to be massive, so the technique is equally applicable for massive $W$ and $Z$ exchanges, as well as their productions if their subsequent decays into (nearly) massless particles are also included in the diagram.

The main idea in the recent development [26] is to write the loop amplitudes in the Schwinger-parameter representation (or the related Feynman-parameter representation) rather than the conventional momentum-space representation. This avoids the presence of off-shell loop momenta and allows the spinor helicity technique to be used (see Sec. 4). This approach is practical because amplitudes in parametric representations can be written down just as easily as amplitudes in momentum representations [28-32] (see Sec. 2).

The main avenue for carrying out string reorganization of multiloop amplitudes [27] is again the Schwinger-parameter representation, though the reason is now different: the Schwinger proper-time parameters turn out to be just the proper time $\tau$ of a superstring in the infinite tension limit. It is therefore not surprising that string reorganization takes place in the parametric rather than the momentum-space framework (see Secs. 3 to 5 ). It turns out that the use of Schwinger representation does not by itself give all the string reorganizations. The use of differential circuit identities, discussed in Appendix A, is essential to obtaining the full result.

In this connection it is crucial to recognize the importance of electric circuit theory (see Appendix A). A Feynman diagram can be regarded as an electric circuit [28-32]: the Schwinger or Feynman parameters are the resistances, the external momenta are the currents flowing in or out of the network, and the internal momenta are currents along the internal lines. The scattering amplitude in parametric representations can be expressed in terms of circuit quantities like power, currents, etc. Knowledge of the relationship between these quantities can lead to simplification of the scattering amplitudes, as will be seen in the rest of this article. Such knowledge includes not only formulas contained in electrical engineering text books, but also differential circuit identities not normally found in the 
literature.

From a field-theoretic point of view, string reorganization accomplishes two things. First, it puts internal and spacetime variables on a somewhat equal footing: they are all flows (current flow, spin flow, color flow, etc.) on the network (see Secs. 3 and 4). Secondly, it succeeds in making local gauge invariance transparent (see Sec. 5 and Appendix D). As a byproduct of the latter, one also sees the possibility of interpreting gauge invariance as a reparametrization invariance in the proper-time variable. This is discussed in Appendix D, between eqs. (D10) and (D11).

\section{Scattering Amplitudes}

The scattering amplitude for a Feynman diagram in $d=4-\epsilon$ dimensions with $n$ vertices, $N$ internal lines, and $\ell$ loops is

$$
A=\left[\frac{-i \mu^{\epsilon}}{(2 \pi)^{d}}\right]^{\ell} \int \prod_{a=1}^{\ell}\left(d^{d} k_{a}\right) \frac{S_{0}(q, p)}{\prod_{r=1}^{N}\left(-q_{r}^{2}+m_{r}^{2}-i \epsilon\right)},
$$

where $k_{a}(1 \leq a \leq \ell)$ are the loop momenta, $q_{r}, m_{r}(1 \leq r \leq N)$ are the momenta and masses of the internal lines, and $p_{i}(1 \leq i \leq n)$ are the conserved external momenta satisfying

$$
\sum_{i=1}^{n} p_{i}=0
$$

For convenience an external momentum $p_{i}$ has been assigned to each vertex. If a vertex is internal, we simply have to set the corresponding $p_{i}=0$ at the end.

The numerator function $S_{0}(q, p)$ contains everything except the denominators of the propagators. Specifically, it is the product of the vertex factors, numerators of propagators, wave functions of the external lines, symmetry factor, and the signs associated with closed



The internal momenta $q_{r}$ are linear combinations of $p_{i}$ and $k_{a}$. In the case of tree diagrams, $k_{a}$ is absent, so

$$
q_{r} \equiv \sum_{i} I_{r i} p_{i}
$$

is given by a linear combination of the external momenta alone. It is this absence of $k_{a}$ that enables the spinor helicity technique to be applied to tree diagrams. See Sec. 4. To use this technique on loop diagrams, it is necessary to get rid of the $k_{a}$-dependence of $q_{r}$ [26]. This can be accomplished by introducing the Feynman parameters $\alpha_{r}$, one per internal line, so the loop integrations can be explicitly carried out. The resulting Feynman-parameter representation reads [30]

$$
\begin{gathered}
A=\int\left[D_{F} \alpha\right] \Delta(\alpha)^{-d / 2} \bar{S}(q, p) \Gamma\left(N-\frac{d \ell}{2}\right)[D(\alpha, p)]^{-N+d \ell / 2}, \\
\int\left[D_{F} \alpha\right] \equiv\left[\frac{\mu^{\epsilon}}{(4 \pi)^{d / 2}}\right]^{\ell}\left(\prod_{r=1}^{N} d \alpha_{r}\right) \delta\left(\sum_{r=1}^{N} \alpha_{r}-1\right),
\end{gathered}
$$




$$
\begin{aligned}
M & \equiv \sum_{r=1}^{N} \alpha_{r} m_{r}^{2} \\
D(\alpha, p) & =M-P(\alpha, p) \\
\bar{S}(q, p) & =\sum_{k \geq 0} i^{k} \frac{\Gamma\left(N-\frac{d \ell}{2}-k\right)}{\Gamma\left(N-\frac{d \ell}{2}\right)} D(\alpha, p)^{k} S_{k}(q, p) .
\end{aligned}
$$

$S_{0}(q, p)$ is the same function as the one appearing in (2.1), except that $q_{r}$ is now given by (2.3), with $k_{a}$ absent and its dependence replaced by the dependence of the coefficients $I_{r i}$ on the $\alpha$ 's. It, along with other quantities in (2.4), can be computed using electric circuit theory. See Appendix A. If the Feynman diagram is regarded as an electric circuit, $\alpha_{r}$ as the resistance of the $r$ th internal line and $p_{i}$ as the current flowing out of the $i$ th vertex, then $q_{r}$ is the current flowing through the $r$ th line and $P$ is the power consumed by the network. Thus

$$
P=\sum_{r=1}^{N} \alpha_{r} q_{r}^{2}=\sum_{i, j=1}^{n} Z_{i j}(\alpha) p_{i} \cdot p_{j}
$$

where $Z_{i j}(\alpha)$ is the impedance matrix. The quantity $\Delta$ is the discriminant appearing in all these circuit quantities.

Suppose the numerator function $S_{0}(q, p)$ is a polynomial in $q$ of degree $e$. Then $S_{k}(q, p)$ is defined to be a polynomial in $q$ of degree $e-2 k$, obtained from $S_{0}(q, p)$ by contracting $k$ pairs of $q$ 's in all possible ways and summing over all the contracted results. The rule for contracting a pair of $q$ 's is:

$$
q_{r}^{\mu} q_{s}^{\nu} \rightarrow-\frac{i}{2} H_{r s}(\alpha) g^{\mu \nu} \equiv q_{r}^{\mu} \sqcup q_{s}^{\nu}
$$

The circuit quantities in (2.4) and (2.6) are given explicitly by the following formulas [30]:

$$
\begin{aligned}
\Delta(\alpha) & =\sum_{T_{1}}\left(\prod^{\ell} \alpha\right) \\
P(\alpha, p) & =\Delta(\alpha)^{-1} \sum_{T_{2}}\left(\prod^{\ell+1} \alpha\right)\left(\sum_{1} p\right)^{2} \\
q_{r}(\alpha, p) & = \pm \Delta(\alpha)^{-1} \sum_{T_{2}(r)} \alpha_{r}^{-1}\left(\prod^{\ell+1} \alpha\right)\left(\sum_{1} p\right) \\
H_{r r}(\alpha) & =-\Delta(\alpha)^{-1} \partial(\alpha) / \partial \alpha_{r}, \\
H_{r s}(\alpha) & = \pm \Delta(\alpha)^{-1} \sum_{T_{2}(r s)}\left(\alpha_{r} \alpha_{s}\right)^{-1}\left(\prod^{\ell+1} \alpha\right), \quad(r \neq s)
\end{aligned}
$$


The meaning of these formulas are as follows. An $\ell$-loop diagram can be made into a connected tree diagram (a '1-tree') by cutting $\ell$ lines, and into a diagram with two disjoint trees (a '2-tree') by cutting $\ell+1$ lines. $\Delta(\alpha)$ is given by the sum over the set $T_{1}$ of all 1-trees so obtained, with the summand equal to the product of the $\alpha$ 's of the cut lines. $\Delta P(\alpha, p)$ is given by the sum over the set $T_{2}$ of all 2 -trees so obtained, with the summand to be the product of the $\alpha$ 's of the cut lines, times the square of the sum of all the external momenta $p_{i}$ attached to one of these two trees. Finally, let $T_{2}(r)$ be the set of all 2-trees in which line $r$ is cut, and such that when the line $r$ is inserted back a 1-tree results, and $T_{2}(r, s)$ be the set of all 2-trees in which lines $r$ and $s$ are cut, and such that when either the line $r$ or the line $s$ is inserted back a 1-tree results. Then $\Delta q_{r}(\alpha, p)$ is given by the sum of all 2-trees in $T_{2}(r)$ with the summand equal to the product of $\alpha$ 's of all the cut lines except the $r$ th, times the sum of all the external momenta $p_{i}$ attached to one of these two trees, and $\Delta H_{r s}(\alpha)$ is given by the sum of all 2-trees in $T_{2}(r, s)$ with the summand equal to the product of $\alpha$ 's of all the cut lines except the $r$ th and the $s$ th. The sign in (2.9) can be determined from the direction of the current flow in an obvious way. The sign in (2.11) is + or - depending on whether lines $r$ and $s$ point to the same tree, or opposite trees.

For example, using eqs. (2.7)-(2.11), the relevant quantities for Fig. 1 can be computed to be

$$
\begin{aligned}
\Delta & =\alpha_{1}+\alpha_{2}+\alpha_{3}+\alpha_{4}, \\
\Delta P & =\alpha_{1} \alpha_{3}\left(p_{1}+p_{3}\right)^{2}+\alpha_{2} \alpha_{4}\left(p_{1}-p_{2}\right)^{2}+\alpha_{1} \alpha_{4} p_{1}^{2}+\alpha_{1} \alpha_{2} p_{2}^{2}+\alpha_{3} \alpha_{4} p_{3}^{2}+\alpha_{2} \alpha_{3} p_{4}^{2} \\
\Delta q_{1} & =\alpha_{2} p_{2}+\alpha_{3}\left(p_{2}+p_{4}\right)+\alpha_{4}\left(p_{2}-p_{3}+p_{4}\right) \\
\Delta H_{13} & =+1 .
\end{aligned}
$$

Other $q_{r}$ and other $H_{r s}$ can be similarly calculated.

Instead of Feynman's method discussed above, one can use Schwinger's proper time formalism. In that case, one writes

$$
\frac{1}{m_{r}^{2}-q_{r}^{2}-i \epsilon}=i \int_{0}^{\infty} d \alpha_{r} \exp \left[-i \alpha_{r}\left(m_{r}^{2}-q_{r}^{2}\right)\right]
$$

and integrates over the internal momenta [see (A19) and (A20)]. The result is [30]

$$
\begin{aligned}
A & =\int\left[D_{S} \alpha\right] \Delta(\alpha)^{-d / 2} S(q, p) \exp [-i\{M-P\}], \quad \text { where } \\
\int\left[D_{S} \alpha\right] & \equiv\left[\frac{(-i)^{d / 2} \mu^{\epsilon}}{(4 \pi)^{d / 2}}\right]^{\ell} i^{N} \int_{0}^{\infty}\left(\prod_{r=1}^{N} d \alpha_{r}\right) \\
S(q, p) & \equiv \sum_{k \geq 0} S_{k}(q, p)
\end{aligned}
$$

with the same functions $M, \Delta, S_{k}, q_{r}$ and $P$ as those appearing in (2.4). These two parametric representations are actually related in a simple way. If for the moment we use $\alpha_{r}^{\prime}$ 
to denote the variables $\alpha_{r}$ in (2.14), and define $\sum_{r} \alpha_{r}^{\prime}=\lambda, \alpha_{r}^{\prime}=\lambda \alpha_{r}$, then $\sum_{r} \alpha_{r}=1$. Carrying out the integration over $\lambda$ in (2.14), eq. (2.4) will be obtained.

It is useful to point out the similarity and the difference between (2.14) and (2.1). The loop-momentum integration in (2.1) is replaced by the Schwinger-parameter integration $\int\left[D_{S} \alpha\right] \Delta^{-d / 2}$ in (2.14); the numerator function $S_{0}(q, p)$ is replaced by $S(q, p)=$ $\sum_{k \geq 0} S_{k}(q, p)$, and the propagator factor $\left(-q_{r}^{2}+m_{r}^{2}-i \epsilon\right)^{-1}$ is replaced by

$$
T\left(q_{r}, m_{r}, \alpha_{r}\right)=\exp \left[-i \alpha_{r}\left(m_{r}^{2}-q_{r}^{2}\right)\right]
$$

which is the time development factor for a particle with momentum $q_{r}$ propagating under the Hamiltonian $H_{o p}=p_{o p}^{2}-m_{r}^{2}$ over a 'proper time' $\alpha_{r}$.

As discussed in the Introduction, a gauge-theory amplitude may contain many many terms. In the language of (2.4) and (2.14), this is reflected in the complexity of computing $S_{k}(q, p)$ and the corresponding $\alpha$-integrals. Methods to simplify their calculations will be the main topic for the rest of this paper. When $S_{k}$ and the other circuit quantities in (2.4) and (2.14) are thus computed, there is still the remaining task of carrying out the $\alpha_{r}$ integrations. In a small number of cases analytical results are known, but in the great majority of situations one would have to resort to numerical methods at that stage. We will not discuss the problem further. If renormalization is required, it has to be carried out in the usual way as well $[32,33]$, and we shall not go into that aspect either.

It would be convenient to consider all scattering processes in a standard form, in which all gluons and photons are outgoing and no antifermions are present. This can be achieved by moving particles from one side of the reaction to another if necessary, thus converting antifermions into fermions and incoming momenta into outgoing, without altering the scattering amplitude $A$. We shall assume in the rest of this article that this has already been done.

\section{Color Flow}

The total scattering amplitude $A_{t o t}$ of a QCD process (obtained by summing all relevant diagrams up to a certain order) depends both on the color and the spacetime quantum numbers of the particles involved. Suppose $\mathcal{C}_{v}$ is a complete set of independent color tensors, then these two dependences can be separated by a color decomposition

$$
A_{t o t}=\sum_{v} \mathcal{C}_{v} a_{v}
$$

This serves to isolate the colorless gauge-invariant subamplitudes $a_{v}$.

The amplitude $A$ for any diagram can be decomposed in a similar way. This is accomplished by decomposing the factors $S_{k}(q, p)$ in $(2.1),(2.4)$, and (2.14):

$$
S_{k}=\sum_{v} \mathcal{C}_{v} s_{k v}
$$


From Feynman rules, it is easy to see that when four-gluon vertices are absent, the color content of $S_{k}$ simply factorizes. In other words, in that case all $s_{k v}$ are proportional to some common $s_{k}$. In $U\left(N_{c}\right)$ gauge theories the proportionality constants are in fact either 0,1 , or -1 ; in $S U\left(N_{c}\right)$ powers of $N_{c}^{-1}$ also appear. If four-gluon vertices are present, then there is a more complicated dependence of $s_{k v}$ on $v$. This will be discussed in Sec. 4.3.

A convenient set of color tensors $\mathcal{C}_{v}$ will be discussed in Sec. 3.1 below. These are the generalizations of the Chan-Paton factors [34] used in the string theory. For a given diagram only some of these color tensors are relevant; a method to determine the relevant ones will be considered in Sec. 3.2.

We shall also assume in the text that we are dealing with a $U\left(N_{c}\right)$ gauge theory. The rules developed below are still valid for $S U\left(N_{c}\right)$ provided linkage of quark lines are always carried through by more than one gluons. Otherwise, $O\left(N_{c}^{-1}\right)$ correction term are present. This and other details will be discussed in Appendix B.

\subsection{Color Tensors}

Let $T^{a}\left(a=0,1, \cdots, N_{c}^{2}-1\right)$ be the generators of $U\left(N_{c}\right)$ in the fundamental (quark) representation, normalized so that

$$
\operatorname{Tr}\left(T^{a} T^{b}\right)=\delta_{a b}, \quad T^{0}=\frac{1}{\sqrt{N_{c}}} \mathbf{1} .
$$

This normalization differs from the usual one by a factor $\frac{1}{2}$ on the rhs. It is convenient for certain purposes but brings grief in others. For $N_{c}=3$, these generators are related to the Gell-Mann $S U(3)$ matrices by $T^{a}=\lambda^{a} / \sqrt{2}$, which means that the structure constant $f^{a b c}$ used here, defined by $\left[T^{a}, T^{b}\right]=i f^{a b c} T^{c}$, also differs from the usual one by a factor $\sqrt{2}$. The net result is that the QCD coupling constant $g$ used in this article is $1 / \sqrt{2}$ of the usual coupling constant.

Let $\left\{a_{1}, \cdots, a_{n}\right\}$ be the color indices for the $n$ (outgoing) gluons, and $\left\{i_{1}, \cdots, i_{m}\right\}$, $\left\{j_{1}, \cdots, j_{m}\right\}$ be respectively the color indices for the outgoing and incoming quarks. A convenient set of color tensors $\mathcal{C}_{v}$ can be obtained as follows.

Let $v$ be any permutation of the set of gluon indices $a_{i}$, written in the cycle form:

$$
v=\left(a_{1}^{\prime} a_{2}^{\prime} \cdots a_{\ell_{1}}^{\prime}\right) \cdots\left(a_{\ell_{c-1}+1}^{\prime} \cdots a_{\ell_{c}}^{\prime}\right)
$$

where $\ell_{i}$ are lengths of the $i$ th cycle, so that $\sum_{i} \ell_{i}=n$, and $c$ is the number of cycles in the permutation $v$. For example, for $n=6$, one such possibility might be $v=$ $\left(a_{3} a_{5}\right)\left(a_{2} a_{1} a_{6}\right)\left(a_{4}\right)$. The color tensors $\mathcal{C}_{v}$ in the absence of fermions can be taken to be

$$
\mathcal{C}_{v}=\operatorname{Tr}\left(a_{1}^{\prime} a_{2}^{\prime} \cdots a_{\ell_{1}}^{\prime}\right) \cdots \operatorname{Tr}\left(a_{\ell_{c-1}+1}^{\prime} \cdots a_{\ell_{c}}^{\prime}\right) .
$$

In (3.5), a shorthand notation is adopted in which a color index $a$ stands for the generator $T^{a}$. Note that a cycle is invariant under cyclic permutation, and so is the trace ' $\mathrm{Tr}$ ', as it should be. 
In the presence of fermions, the construction of $\mathcal{C}_{v}$ is more complicated. In this case they are of the form

$$
\mathcal{C}_{v}=\prod_{k=1}^{m}\left(T^{b_{k}}\right)_{i_{k} j_{k}^{\prime}} C_{v^{\prime}}^{0},
$$

where $\left\{j_{1}^{\prime}, \cdots, j_{m}^{\prime}\right\}$ is a permutation of $\left\{j_{1}, \cdots, j_{m}\right\}$, and $\left\{b_{1}, \cdots, b_{m}\right\}$ together with the color indices in $v^{\prime}$ form a permutation of the gluon indices $\left\{a_{1}, \cdots, a_{n}\right\}$. The symbol $C_{v}^{0}$ stands for the tensor $\mathcal{C}_{v}$ in $(3.5)$.

\subsection{Feynman diagrams}

The relevant color tensors $\mathcal{C}_{v}$ appearing in a given Feynman diagram can be read off from the color paths of the diagram [26]. A formal definition of the color paths is given immediately below, but it is perhaps far easier to look at the examples first before examining the formal definitions.

A color path is a continuous path along the lines of the diagram drawn in such a way that

1. every quark line is traversed at most once and every gluon line as well as every ghost line is traversed at most twice;

2. paths along quark lines are directed along the arrow of the quark lines. Paths along gluon lines can move in either directions;

3. at a quark-gluon vertex junction, path along a quark line turns to follow a gluon line and vice versa;

4. a path may be open or closed. If open, it must start at an incoming quark and end at an outgoing quark. In particular, a path cannot end at an external gluon line, so when it comes to the end of such a line it must reverse and retrace its path.

A color covering of the diagram is a collection of color paths so that every quark line in the diagram is traversed exactly once, and that every gluon line and every ghost line in the diagram is traversed exactly twice.

5. for each color covering, there is a predetermined order of paths through every threegluon, four-gluon, or ghost-gluon vertex junction, as explained below.

At each three-gluon or ghost-gluon vertex, three lines with colors $a, b, c$ are involved. There are two ways to cyclically order these three colors: $(a b c)$ and $(a c b)$. Each ordering fixes an order of the paths passing through this vertex. For example, in the ordering $(a b c)$, the path leading into the vertex on line $a$ (respectively $b, c$ ) must exit the vertex on line $b$ ( respectively $c, a$ ). Different orderings correspond to different paths and different color coverings. Similarly, at a four-gluon vertex, there are six ways to cyclically order the four colors $a, b, c, d$ and consequently the corresponding paths through the vertex. The ordering $(a d c b)$ for example specifies that the path enetering the vertex along line $a$ ( respectively $b, c, d)$ must leave along line $d($ respectively $a, b, c)$.

Let us look at some concrete examples. In Fig. 1, color paths $\left(p_{2} q_{2} q_{3} q_{4} p_{1}\right) \equiv v_{1}$ and 
$\left(p_{4} q_{2} q_{1} q_{4} p_{3}\right) \equiv v_{2}$ together form a covering of the Feynman diagram. In these notations, momenta read from right to left are used to label paths.

An example involving the presence of three- and four-gluon vertices is shown in Fig. 2. In that case there are several color coverings, one of which being $\left(p_{2} q_{7} q_{8} q_{5} q_{11} q_{9} q_{1} q_{10}\right.$ $\left.q_{12} p_{3}\right) \equiv v_{1},\left(p_{4} p_{5} p_{5} q_{6} q_{8} p_{7} p_{7} q_{7} q_{2} q_{9} q_{10} p_{1}\right) \equiv v_{2}$, and $\left(q_{4} p_{6} p_{6} q_{3} q_{12} q_{11}\right) \equiv v_{3}$. In this case, the ordering at the three gluon vertex is $\left(q_{7} q_{8} p_{7}\right)$ (momenta are used here to represent the corresponding colors) and the ordering at the four-gluon vertex is $\left(q_{11} q_{9} q_{10} q_{12}\right)$.

To each color path $v_{a}$ is associated a color tensor $\mathcal{C}_{v_{a}}$. If $v_{a}$ is closed, then $\mathcal{C}_{v_{a}}=\operatorname{Tr}(\cdots)$, where $\cdots$ stands for a succession of the color indices of the external gluons, written from right to left in the order that the path encounters. If $v_{a}$ is open, leading from an incoming quark line with color index $j$ to an outgoing quark line with color index $i$, then $\mathcal{C}_{v_{a}}=(\cdots)_{i j}$. If $\left\{v_{1}, v_{2}, \cdots, v_{u}\right\}$ is a path-covering of the Feynman diagram, then the total color factor for the amplitude $A$ is $\mathcal{C}_{v}=\prod_{a=1}^{u} \mathcal{C}_{v_{a}}$. Notice that these tensors are of the form (3.6).

For example, for Fig. 1,

$$
\mathcal{C}_{v_{1}}=(\mathbf{1})_{i j}, \quad \mathcal{C}_{v_{2}}=(\mathbf{1})_{i^{\prime} j^{\prime}},
$$

and for the color covering of Fig. 2 chosen before,

$$
\mathcal{C}_{v_{1}}=(\mathbf{1})_{i j^{\prime}}, \quad \mathcal{C}_{v_{2}}=(a c)_{i^{\prime} j} \equiv\left(T^{a} T^{c}\right)_{i^{\prime} j}, \quad \mathcal{C}_{v_{3}}=\operatorname{Tr}(b) \equiv \operatorname{Tr}\left(T^{b}\right) .
$$

This association of color tensors with color paths is derived for $U\left(N_{c}\right)$ colors, but it remains valid for $S U\left(N_{c}\right)$ provided pairs of quark lines, when linked, are always carried out by more than one gluon lines at a time. For example, (3.7) is not valid for $S U\left(N_{c}\right)$ because each of the two linkages of the two quark lines is carried out only by one gluon line. On the other hand, (3.8) is valid for $S U\left(N_{c}\right)$ as well because both the linkages contain more than one gluon lines: they are linked by two and four internal gluon lines respectively.

\section{Spin Flow}

The factors $s_{k v}$ in (3.2) contains various scalar products and fermion matrix elements like $\bar{u} \Gamma u$. Here $\Gamma$ is a scalar function of the Dirac matrices $\gamma$, the external momenta $p_{i}$, and the gluon/photon polarization vectors $\epsilon_{i}$, and $u, \bar{u}$ are the fermion wave functions. From these expressions, one must reduce the algebra to express the amplitude $|A|$ in terms of measurable quantities, i.e., the scalar products of the external momenta and the helicities of external particles. In the case of $S_{k}$ or $s_{k v}$, Feynman parameters will be involved as well. This task of algebraic reduction is not at all easy for gauge theories because of the rather complicated dependences of $u$ and $\epsilon$ on the measurable quantities, and because of the involvement of the non-commutative Dirac algebra. Fortunately, for high energy processes where the masses of the external particles can be ignored, such calculations can be immensely simplified by using the spinor helicity technique [1-21]. The main purpose of this section is to discuss how this technique can be applied to obtain $S_{k}$. We shall assume in the following $d=4$. If $d \neq 4$, the usual complication of $\gamma_{5}$ and of the polarization vector [36] arise and they have to be dealt with in the usual way. 
The main idea of this technique is explained in Sec. 4.1, leaving the detailed algebra and justification to Appendix C. Its application to spinor QED and QCD is explained in Secs. 4.2 and 4.3, via a graphical technique making use of spinor paths. Spinor paths are very similar to color paths owing to the resemblance between the color algebra and the Dirac algebra. Yet in detail the two kinds of paths are different because of the different dependences of the Feynman rules on color and spin.

\subsection{Spinor Helicity Technique}

The involvement of Dirac algebra can be traced back to a mixing of helicity and chiral eigenstates. An external (massive) fermion carries a definite helicity, but not a definite chirality. On the other hand, it is chirality that is conserved at QED and QCD vertices. This forces a mixing of helicity states whenever interactions take place, which brings about a multichannel problem described by the $4 \times 4$ Dirac matrices. However, if the external fermion is massless, or can be regarded as such in a high energy process, then helicity and chirality are essentially the same, no mixing of states need to occur, the four-channel problem is reduced to a one-channel problem and the complicated Dirac algebra can thus be avoided. This is the basic idea of the spinor helcity technique [1-21].

This technique is also useful for spin-1 particles, because kinematically a spin-1 particle may be thought of as a composite of two spin- $\frac{1}{2}$ particles. Moreover, the polarization vector of a gauge particle is gauge dependent, so in principle it is possible to simplify calculations by a suitable gauge choice. This turns out to be so in the spinor helicity technique.

Unless otherwise specified, it will be assumed from now on that all external momenta $p_{i}$ are massless. The massless spin- $\frac{1}{2}$ wave functions $u_{\lambda}\left(p_{i}\right)$ and $\bar{u}_{\lambda}\left(p_{i}\right)$ will be written respectively as $\left|p_{i} \lambda\right\rangle$ and $\left\langle p_{i} \lambda\right|$, where $\lambda= \pm 1$ is (twice) the helcity of the fermion. Using the fact that these are also $\gamma_{5}$ eigenstates, one concludes immediately that

$$
\left\langle p_{i} \pm \mid p_{j} \pm\right\rangle=0
$$

The non-zero matrix elements are conventionally written as

$$
\left\langle p_{i} p_{j}\right\rangle \equiv\left\langle p_{i}-\mid p_{j}+\right\rangle, \quad\left[p_{i} p_{j}\right] \equiv\left\langle p_{i}+\mid p_{j}-\right\rangle
$$

whose normalization and charge conjugation properties can be chosen so that

$$
\begin{aligned}
\left\langle p_{i} p_{j}\right\rangle & =-\left\langle p_{j} p_{i}\right\rangle, \\
{\left[p_{i} p_{j}\right] } & =-\left[p_{j} p_{i}\right], \\
\left\langle p_{i} p_{j}\right\rangle\left[p_{j} p_{i}\right] & =2 p_{i} \cdot p_{j} \\
{\left[p_{j} p_{i}\right] } & =\operatorname{sign}\left(p_{i} \cdot p_{j}\right)\left\langle p_{i} p_{j}\right\rangle^{*} .
\end{aligned}
$$

See Appendix $\mathrm{C}$ for the derivation of these and other related formulas.

The polarization vectors for an outgoing photon/gluon of momentum $p$ and helicity \pm 1 can be chosen to be

$$
\epsilon_{ \pm}^{\mu}(p, k)= \pm \frac{\left\langle p \pm\left|\gamma^{\mu}\right| k \pm\right\rangle}{\sqrt{2}\langle k \mp \mid p \pm\rangle}
$$


where $k$ is an arbitrary massless momentum known as the reference momentum. Polarization vectors with different $k$ 's are related by gauge transformations (see (C21)). There are two advantages with this form of the polarization vector: it is expressed in terms of spinor notations so the spinor helicity technique can be used; it is also symmetrical in $p$ and $k$ so that $k \cdot \epsilon_{ \pm}(p, k)=0$ as well as $p \cdot \epsilon_{ \pm}(p, k)=0$, making it possible to simplify the calculation by a proper choice of $k$.

It turns out that all matrix elements of the Dirac matrices and all expressions involving the polarization vectors can be reduced to the bracket quantities in (4.2), which according to (4.3)-(4.6), are essentially the square roots of the dot momenta. In this way the objective of expressing everything in terms of measurable quantities is realized.

Graphical methods can be designed to read off $S_{k}$ directly from the Feynman diagram. This will be illustrated in the next two sections for massless QED and QCD, but other rules can be formulated to cover more complicated situations [26]. Feynman gauge will be used throughout this paper for gauge propagators .

\subsection{Spinor QED}

In order to describe how $S_{0}(q, p)$ is computed graphically, it is necessary to discuss what a spinor path and a spin factor are. Again, it might be easier to follow the illustrative examples before returning to the formal definitions immediately below.

Consider first diagrams with no external photons present. A spinor path is a continuous path along the lines of the diagram drawn in such a way that

1. every electron line is traversed at most once and every photon line as well as every ghost line is traversed at most twice;

2. a path along an electron line must travel in the same (opposite) direction of the previous electron line the path went through if these two electron lines carry opposite (the same) helicities. Paths along internal photon lines can move in either directions;

3. a path along an electron line must turn to follow an internal photon line, and vice versa, when an electron-photon vertex is encountered;

4. a path may be open or closed. If open, it must start and end at external electron lines.

In other words, in the absence of photons, a spinor path in QED is almost identical to a color path in QCD, except for the fact that the spinor path can have both orientations along an electron line depending on the helicities involved. We will see that more differences will emerge when external photons are present, and when other vertices are encountered in the case of QCD.

A spinor covering of the diagram is a set of spinor paths so that every electron line in the diagram is traversed exactly once and every photon line exactly twice by these paths.

For example, Fig. 3 is covered by three spinor paths, $\left(p_{2} q_{5} q_{1} q_{7} p_{3}\right) \equiv A,\left(p_{4} q_{6} q_{3} q_{5} p_{1}\right) \equiv$ $B$, and $\left(q_{4} q_{6} q_{2} q_{7}\right) \equiv C$, in which the paths are labelled by the momenta of the lines they pass through, to be read from right to left. Paths A and B are open, and path $\mathrm{C}$ is closed. 
It is easy to see from the above list that every electron line $\left(p_{i}, q_{i}, 1 \leq i \leq 4\right)$ is traversed exactly once and every internal photon line $\left(q_{5}, q_{6}, q_{7}\right)$ is traversed exactly twice. Note that if the upper electron line carried negative helicity, then the diagram will be covered by only two paths $A^{\prime}$ and $B^{\prime}:\left(p_{4} q_{6} q_{2} q_{7} p_{3}\right) \equiv A^{\prime}$ and $\left(p_{2} q_{5} q_{1} q_{7} q_{4} q_{6} q_{3} q_{5} p_{1}\right) \equiv B^{\prime}$.

To each spinor path $\mathrm{A}$ is associated a spin factor $\mathcal{G}_{A}$ obtained by pairing consecutive momenta along the path, alternately with brackets $\langle\cdots\rangle$ and $[\cdots]$. In this pairing, the momentum $q_{r}$ of an internal fermion always occurs twice, as in $\left[\cdot q_{r}\right]\left\langle q_{r} \cdot\right\rangle$, or in $\left\langle\cdot q_{r}\right\rangle\left[q_{r} \cdot\right]$, but the momentum of any internal gluon line is to be omitted. For an open path, one can start the pairing process either from the end of the outgoing electron, or from the end of the incoming electron. In the former case, the pairing proceeds from left to right, starting with a $\langle\cdots([\cdots)$ when the outgoing electron has a negative (positive) helicity. In the latter case, the pairing proceeds from right to left, starting with a $\cdots\rangle(\cdots])$ when the incoming electron has a positive (negative) helicity. If the path is closed, then one can start at any point along the path, but both helicities should be used and a sum taken.

For example, the spin factors for Fig. 3 are

$$
\begin{aligned}
& \mathcal{G}_{A}=\left[p_{2} q_{1}\right]\left\langle q_{1} p_{3}\right\rangle \\
& \mathcal{G}_{B}=\left[p_{4} q_{3}\right]\left\langle q_{3} p_{1}\right\rangle \\
& \mathcal{G}_{C}=\left[q_{4} q_{2}\right]\left\langle q_{2} q_{4}\right\rangle+\left\langle q_{4} q_{2}\right\rangle\left[q_{2} q_{4}\right]=4 q_{2} \cdot q_{4} .
\end{aligned}
$$

If instead the upper electron has negative helicity, then

$$
\begin{aligned}
\mathcal{G}_{A^{\prime}} & =\left\langle p_{4} q_{2}\right\rangle\left[q_{2} p_{3}\right], \\
\mathcal{G}_{B^{\prime}} & =\left[p_{2} q_{1}\right]\left\langle q_{1} q_{4}\right\rangle\left[q_{4} q_{3}\right]\left\langle q_{3} p_{1}\right\rangle .
\end{aligned}
$$

The momentum pairs appearing in (4.2) are massless, but in the pairings above offshell momenta $q_{r}$ appear. In their presence the brackets are defined recursively as follows:

$$
\begin{aligned}
\left\langle p_{i} q_{r}\right\rangle\left[q_{r} p_{j}\right] & \equiv \sum_{k} I_{r k}\left\langle p_{i} p_{k}\right\rangle\left[p_{k} p_{j}\right], \\
{\left[p_{i} q_{r}\right]\left\langle q_{r} p_{j}\right\rangle } & \equiv \sum_{k} I_{r k}\left[p_{i} p_{k}\right]\left\langle p_{k} p_{j}\right\rangle,
\end{aligned}
$$

where $I_{r k}$ are the coefficients appearing in (2.3). Note that only bilinear combinations as appearing on the left hand side of (4.10) are defined, and this will always be the case for the brackets appearing in the spin factors $\mathcal{G}_{A}$. Single unpaired $\left\langle q q^{\prime}\right\rangle$ or $\left[q q^{\prime}\right]$ for off-shell $q, q^{\prime}$ remain undefined. Note also that if $q_{r}=\kappa p_{k}$ for some constant $\kappa$ and some massless momentum $p_{k}$, then (4.10) is perfectly consistent with eq. (C4).

So far we have considered diagrams without external photons. In their presence, spinor paths and spin factors can be calculated by modifying the diagram in the following way. Attach a short fermion line to every external photon line and assign the following characteristics to these artificial fermions. The helicity of the artificial fermion is equal to 
the helicity of the photon, the momentum of the incoming artificial fermion is equal to the reference momentum of the photon, and the momentum of the outgoing artificial fermion is equal to the actual momentum of the photon. For example, the modified diagram for Fig. 4 is given in Fig. 5.

The modified diagrams should now be considered as a Feynman diagram without external photon lines, and their spinor paths as well as their spin factors $\mathcal{G}_{A}$ should be calculated just as before. For example, the spin factors for Figs. 4 or 5 are

$$
\begin{aligned}
\mathcal{G}_{A} & =\left[p_{2} q_{1}\right]\left\langle q_{1} p_{3}\right\rangle, \\
\mathcal{G}_{B} & =\left[p_{4} p_{5}\right], \\
\mathcal{G}_{C} & =\left\langle k_{5} q_{9}\right\rangle\left[q_{9} q_{3}\right]\left\langle q_{3} p_{1}\right\rangle, \\
\mathcal{G}_{D} & =\left\langle p_{6} q_{6}\right\rangle\left[q_{6} q_{5}\right]\left\langle q_{5} q_{8}\right\rangle\left[q_{8} k_{6}\right],
\end{aligned}
$$

where $k_{5}, k_{6}$ are respectively the reference momenta for the photons with momenta $p_{5}$ and $p_{6}$. In actual calculations, $k_{5}, k_{6}$ are usually chosen to be one of the $p_{i}$ 's.

In terms of these spin factors, $S_{0}(q, p)$ in $(2.4)$ is given by

$$
S_{0}(q, p)=\mathcal{N G}
$$

where $\mathcal{G}=\prod_{A} \mathcal{G}_{A}$ is the product of the spin factors $\mathcal{G}_{A}$ over all the paths $A$ in a spinor covering. If the diagram should have more than one spinor coverings (which happens in QCD but never in QED), then a sum over all the coverings should be taken as well.

The factor $\mathcal{N}$ for a diagram with a symmetry factor $S_{s y m}, \ell_{F}$ fermion loops, $n_{\gamma}$ external and $N_{\gamma}$ internal photon lines is

$$
\mathcal{N}=(\sqrt{2} e)^{n_{\gamma}+2 N_{\gamma}}(-1)^{\ell_{F}} S_{\text {sym }}^{-1} \prod_{a=1}^{n_{\gamma}} \mathcal{F}_{a}
$$

where

$$
\mathcal{F}_{a}= \begin{cases}+1 /\left\langle k_{a} p_{a}\right\rangle, & \left(\lambda_{a}=+1\right) \\ -1 /\left[k_{a} p_{a}\right], & \left(\lambda_{a}=-1\right),\end{cases}
$$

with $p_{a}, k_{a}, \lambda_{a}$ being respectively the actual momentum, the reference momentum, and the helicity of the ath external photon.

Similar graphical rule can be developed to calculate $S_{k}$. This involves shrinking the contracted lines and making new links according to (2.6).

\subsection{QCD}

The QCD colorless factors $s_{0 v}$ in (2.14) is like the factor $S_{0}$ in QED, if we make the obvious substitution of quarks and gluons for electrons and photons. It is given by the right-hand side of (4.12), with $\mathcal{N}$ and $\mathcal{F}_{a}$ given by (4.13) and (4.14). The factor $\mathcal{G}$ is now dependent on the color path $v$ if four-gluon vertices are present, and it is equal to

$$
\mathcal{G}=\sum \prod_{A} \mathcal{G}_{A v},
$$


where the sum is taken over all the spinor coverings of the diagram. The spinor factor $\mathcal{G}_{A v}$ depends on the color path $v$ only when multi-gluon or ghost-gluon vertices are present. Otherwise, $\mathcal{G}_{A v}$ is calculated exactly like QED.

Before discussing how spinor paths are constructed in the presence of these nonabelian vertices, let us first digress to discuss (color-)oriented vertices [26] in the background Feynman gauge [37]. These are shown in Fig. 6.

A background gauge is one in which the gauge-fixing depends explicitly on a background Yang-Mills field from which external gluon lines are extracted. As a result, vertices containing external gluon lines (indicated by a circled 'A' in Fig. 6) have different Feynman rules than vertices without. Furthermore, extra ghost-gluon vertices appear, as shown in Fig. 6, where a thin solid line represents a gluon $(g)$, a thick solid line a quark $(q)$, and a dashed line a ghost $(G)$. A background Feynman gauge is one in which Feynman gauge is used in the gluon propagators.

An oriented vertex differs from an ordinary vertex in that the lines in the vertex diagram are ordered in a clockwise order, and the vertex Feynman rules depend on the line orderings. It is the appropriate vertex to use when color factors are taken to be the Chan-Paton tensors of Sec. 3.1 rather than the usual ones. Their Feynman rules can be derived from the ordinary Feynman rules by using eqs. (B3) and (B4). In particular, those in the background Feynman gauge can be derived this way from Fig. 1 of the first paper in [37] by setting $\alpha=1$.

The Feynman rules for Fig. 6 are indicated below the diagrams, and they should be interpreted in a particular way. A line with a heavy dot at the end is a dislodged line. The Feynman-rule factors for diagrams with dislodged vertices are always vectors, whose component is indicated by the Lorentz index of the dislodged line. A continuous gluon line signifies a $g$.. factor. In Fig. 6 the color and coupling-constant factors are understood: there should always be a coupling constant $g$ for every cubic diagram and a $g^{2}$ for every quartic diagram. The color factor for Fig. $6(\mathrm{t})$ is $T^{a}$, and that of any other diagram is $\operatorname{Tr}\left[a_{1} a_{2} a_{3}\left(a_{4}\right)\right]$, where $a_{1}, a_{2}, a_{3},\left(a_{4}\right)$ are the color indices of the $g$ or $G$ lines read in clockwise order. Finally, as mentioned before, an external gluon line is indicated by a circled 'A'. The gluon lines without a circled 'A' can be either an external or an internal gluon line, unless such a configuration is already explicitly represented by a diagram in Fig. 6 with circled 'A's.

To give some examples, let the (outgoing momentum, Lorentz index, color index) of line 1 be $\left(q_{1}, \alpha, a\right)$, that of line 2 be $\left(q_{2}, \beta, b\right)$, etc. An external momentum will be designated as $p_{i}$ rather than $q_{i}$. Fig. 6(b) is a vertex for one external and two internal gluon lines, with factor $g \operatorname{Tr}(a b c)\left(q_{2}-q_{3}\right)_{\alpha} g_{\beta \gamma}$. Similarly, Fig. 6(e) is a vertex for four internal gluon lines, with vertex factor $g^{2} \operatorname{Tr}(a b c d)(+2) g_{\alpha \gamma} g_{\beta \delta}$.

Note that all topologically different permutations must be taken into account. For example, in the usual four-gluon vertex, there are six terms with a color factor like $f^{a b e} f^{c d e}$ in each. Using eq. (B4), this generates 24 terms. All these 24 terms are summarized in Figs. 6(e) and 6(f) as follows. There are six possible permutation of color indices in each diagram. For a fixed permutation, there are two different diagrams of type 6(f), e.g., 
$\operatorname{Tr}(a b c d) g_{\alpha \beta} g_{\gamma \delta}$ and $\operatorname{Tr}(a b c d) g_{\delta \alpha} g_{\beta \gamma}$. The factor of +2 is $6(\mathrm{e})$ also represents two terms. Altogether there are thus $(2+2) \times 6=24$ terms, as it should be.

The main advantage of the background-gauge is that in Figs. 6(c) and 6(d), where an internal line is dislodged, the vertex factor depends only on the external momentum. This simplifies matters in loop calculations because otherwise internal momenta are involved, they depend on the Feynman (Schwinger) parameters and must be calculated using (2.9), and they are also subject to contractions (2.6). For low order calculations with many external photons/gluons, this simplification far outweighs the complications brought in by the presence of the extra vertices in Fig. 6.

If ordinary (not background) gauges are used, then there should be no distinction between internal and external gluon lines. All diagrams containing a circled 'A' in Fig. 6 can be put to zero. In particular, there is only one triple-gluon vertex, that of Fig. 6(a).

From Fig. 6 it is possible to deduce how spinor paths and spinor coverings are constructed in the presence of the extra QCD gluon vertices. First modify the $(g g g)$ vertices in a Feynman diagram by dislodging one of the three gluon lines. There are obviously three different ways of doing so and these correspond to different spinor paths. Next, pair up the four gluon lines in a $(g g g g)$ vertex into two pairs, similar to what is shown in Figs. 9(e)-(h). There are again three ways of doing so and these again correspond to different spinor paths.

The rules for constructing a spinor path are identical to the rules in QED, with the obvious replacement of electrons by quarks and photons by gluons. The following addition rule tells us what to do when a non-abelian vertex is encountered:

5. a dislodged gluon line should be regarded as if it were an external gluon line; a paired gluon line should be treated as a single continuous line.

The spinor factor $\mathcal{G}_{A v}$ in (4.15) is determined in exactly the same way as in the QED case, but with the following addendum. The artificial fermion line attached to a dislodged gluon line should carry initial and final momenta equal to the vector given below the appropriate diagram in Fig. 6. The helicity of the artificial fermion is immaterial and can be chosen to be either. Additional numerical factors appearing in Fig. 6 and the appropriate number of coupling constants should also be incorporated into $\mathcal{G}_{A v}$. Note that there is very little coupling between the color path $v$ and the spinor path $A$ : only a sign and perhaps a numerical factor.

Now examples. There is only one color covering each for Figs. 1 and 3 , and the $\mathcal{G}$ factors were given in (4.8) and (4.11) respectively.

Consider Fig. 2 with the color path discussed in Sec. 3.2. Fig. 7 indicates a spinor covering for a possible helicity configuration, where $k_{i}$ are the reference momenta of the external gluons with actual momenta $p_{i}$. The spinor factors in the background gauge are

$$
\begin{aligned}
& \mathcal{G}_{A}=\left[p_{2} k_{7}\right], \\
& \mathcal{G}_{B}=\left\langle p_{7} q_{2}\right\rangle\left[q_{2} q_{5}\right]\left\langle q_{5}\left(2 p_{7}\right)\right\rangle, \\
& \mathcal{G}_{C}=\left[\left(2 p_{7}\right) q_{6}\right]\left\langle q_{6} k_{5}\right\rangle,
\end{aligned}
$$




$$
\begin{aligned}
\mathcal{G}_{D} & =\left[p_{5} p_{4}\right] \\
\mathcal{G}_{E} & =\left\langle p_{3} p_{1}\right\rangle \\
\mathcal{G}_{F} & =(-1)\left\langle p_{6} q_{3}\right\rangle\left[q_{3} q_{1}\right]\left\langle q_{1} q_{4}\right\rangle\left[q_{4} k_{6}\right] .
\end{aligned}
$$

\section{String-like Formulas}

A one-loop $n$-photon/gluon amplitude can be computed using superstring and first quantized techniques [22-25]. The result resembles (2.14), but the numerator function $S(q, p)$ differs from what one would get by using $(2.6)-(2.11)$ directly. The purpose of this section is to show that these stringlike formulas can also be obtained by purely fieldtheoretic means [27], and in this way they can be generalized to multiloop amplitudes and to all processes.

Let $a$ be a cubic vertex in scalar QED, with an external photon line as shown in Fig. 8. Its vertex factor is

$$
C_{a}=e \epsilon_{a} \cdot\left(q_{a^{\prime}}+q_{a^{\prime \prime}}\right),
$$

where $\epsilon_{a}$ is the polarization vector of the outgoing photon. What the stringlike reorganization achieves, see Sec. 5.1, is to replace these factors and their contractions by derivatives wrt the Schwinger parameters. The advantage of this new form, compared to the direct use of (2.9)-(2.11), is that different terms are connected by derivatives so that integration by parts $[22-25]$ can be used to relate them.

For spinor QED, the vertex factor is changed to

$$
F_{a}=e \epsilon_{a} \cdot \gamma,
$$

and there are additional momentum dependence from the spinor propagators. By using the Gordon identity, $F_{a}$ can be partially converted to $C_{a}$ so the stringlike formulas for scalar QED can be applied. See Sec. 5.2. However, Gordon identity changes chirality-conserving amplitudes to chirality-violating amplitudes, so this change generally does not mesh well with the spinor helicity technique.

For QCD, a three-gluon vertex has a term resembling (5.1), but it also has two other terms. In the background gauge discussed in Sec. 4.3, the other two terms only depend on the external momenta, so the stringlike reorganization for scalar QED is once again applicable here. See Sec. 5.3.

From a purely field-theoretical point of view, these new formulas can be motivated by, and derived from, the desire to restore manifest local gauge invariance. To do so considerable knowledge of the electric circuit theory is required. These details will be supplied in Appendices A and D.

For topological reasons, these stringlike reorganizations can be carried out only at external vertices. Whether other simplifications exist for internal vertices is presently unknown.

\subsection{Scalar QED}


The scalar particles considered here are allowed to interact 'strongly' among themselves in any non-derivative manner. Their interactions with the photon field $A_{\mu}$ are given by the cubic vertex $C^{\mu} A_{\mu}$ of Fig. 8 and the quartic (seagull) vertex $Q^{\mu \nu} A_{\mu} A_{\nu}$ of Fig. 9, where

$$
\begin{aligned}
C^{\mu} & =e\left(q^{\prime \prime}+q^{\prime}\right)^{\mu}, \\
Q^{\mu \nu} & =e^{2} g^{\mu \nu} .
\end{aligned}
$$

The numerator of photon propagator in covariant gauges is

$$
-g^{\mu \nu}+\xi p^{\mu} p^{\nu} / p^{2}
$$

Unless otherwise specified, the Feynman gauge $\xi=0$ will be used throughout.

Consider a QED diagram with $n_{e}$ external vertices, of which $n_{3}$ are cubic vertices of the type (5.1), with outgoing photon momenta $p_{a}$ and polarization $\epsilon_{a}$. Let $S_{0}^{e x t}(q, p)$ be the product of these $n_{3}$ vertex factors, and the numerator function be $S_{0}(q, p)=$ $S_{0}^{\text {ext }}(q, p) S_{0}^{\text {int }}(q, p)$. Then the scattering amplitude is given by $(2.14)$ to be

$$
A=\int\left[D_{S} \alpha\right] \Delta^{-d / 2} S(q, p) \exp [-i(M-P)]
$$

which turns out to equal to

$$
A=\int\left[D_{S} \alpha\right] \Delta^{-d / 2}\left\{S^{i n t}(\tilde{q}, p) \exp [-i(M-\tilde{P})]\right\}_{M L}
$$

where $S^{i n t}=\sum_{k} S_{k}^{i n t}$ is the total numerator factor with the $n_{3}$ vertices removed. If $q_{r}$ and $P$ are given by $(2.3)$ and $(2.5), a, b$ are external vertices like Fig. 8 , then

$$
\begin{aligned}
\tilde{P} & =\sum_{a, b=1}^{n_{e}}\left(p_{a}+i e \eta_{a} \epsilon_{a} \partial_{a}\right) \cdot\left(p_{b}+i e \eta_{b} \epsilon_{b} \partial_{b}\right) Z_{a b}(\alpha) \\
\tilde{q}_{r} & =\sum_{a=1}^{n_{e}}\left(p_{a}+i e \eta_{a r} \epsilon_{a} \partial_{a}\right) I_{r a}(\alpha), \quad \text { with } \\
\partial_{a} & \equiv \frac{\partial}{\partial \alpha_{a^{\prime}}}-\frac{\partial}{\partial \alpha_{a^{\prime \prime}}}
\end{aligned}
$$

$\eta_{a}$ is defined to be 1 for one of these $n_{3}$ vertices and 0 otherwise. Similarly,

$$
\eta_{a r}= \begin{cases}1, & \text { if } \eta_{a}=1 \text { and line } r \text { is not adjacent to vertex } a \\ 2, & \text { if } \eta_{a}=1 \text { and } a \text { is at one end of the line } r \\ 0, & \text { if } \eta_{a}=0\end{cases}
$$


in the $n_{3}$ polarization vectors $\epsilon_{a}$ should be retained when the curly bracket is expanded. 
Eq. (5.7) is the result of the stringlike reorganization. It is valid provided the impedance matrix $Z_{a b}$ is given in the diagonal scheme of (A16), where $Z_{a a}=0$ for every $a$. In this scheme (see Appendix A for more details), the impedance matrix can be computed using the graph-theoretical formula [see (A18)]

$$
Z_{a b}=-\frac{1}{2} \sum_{T_{2}^{a b}} \prod^{\ell+1} \alpha, \quad(a \neq b),
$$

where $T_{2}^{a b}$ is the set of all 2-trees with vertices $a$ and $b$ belonging to two separate 1-trees.

Sting-like organization replaces all external cubic electromagnetic vertices by momentum shifting as in (5.8) and (5.9). The $\alpha$-dependence of the original and the shifted terms differ only by derivatives $\partial_{a}$ and $\partial_{b}$, so they can be converted into one another by an integration by parts. This can lead to simplification in calculations [22-25]. In the special case of a one-loop $n$-photon amplitude, this formula was first obtained from string calculations. The formula (5.7) however is valid for multiloop amplitudes, and for all scattering processes.

Eq. (5.7) can be derived from an attempt to understand local gauge invariance. Its derivation and the question of manifest gauge invariance will be taken up in Appendix D.

\subsection{Spinor QED}

The vertex in spinor QED is given by (5.2). The main idea in stringlike reorganization is to use the Gordon identity to convert it partially to the scalar vertex (5.1), so that the formulas in Sec 5.1 can be used once again. The Gordon identity on mass shell is well known to be

$$
\bar{u}_{\lambda^{\prime}}\left(p^{\prime}\right) \gamma^{\mu} u_{\lambda}(p)=\frac{1}{2 m} \bar{u}_{\lambda^{\prime}}\left(p^{\prime}\right)\left[\left(p^{\prime}+p\right)^{\mu}+i \sigma^{\mu \nu}\left(p^{\prime}-p\right)_{\nu}\right] u_{\lambda}(p) .
$$

A similar off-shell formula exists [27] and will be explained in Appendix E. This formula allows the electromagnetic vertex $F^{\mu} A_{\mu}=e \gamma^{\mu} A_{\mu}$ to be replaced by a sum of the scalar QED vertices, the cubic vertex $C^{\mu} A_{\mu}=e\left(q^{\prime \mu}+q^{\prime \prime} \mu\right) A_{\mu}$, the seagull vertex $Q^{\mu \nu} A_{\mu} A_{\nu}=$ $e^{2} g^{\mu \nu} A_{\mu} A_{\nu}$, and the magnetic-moment vertex

$$
S^{\mu} A_{\mu}=-i e A_{\mu} \sigma^{\mu \nu} p_{\nu}
$$

provided the spinor propagators $(m-\gamma q)^{-1}$ are simultaneously replaced by the scalar propagators $\left(m^{2}-q^{2}\right)^{-1}$. In addition, a factor $(2 m)^{-1}$ should be added to every pair of external fermion lines, and a factor $\frac{1}{2}$ to every fermion loop.

In the original form, only helicity-conserving matrix elements survive in the $m \rightarrow 0$ limit. See Sec. 4.2. In the modified form, propagators no longer contribute to $S_{0}(q, p)$, and the new vertices $C, Q, S$ all contain an even number of Dirac $\gamma$-matrices. Hence helicityconserving matrix elements tend to zero in the $m \rightarrow 0$ limit, which is why the extra factors $(2 m)^{-1}$ are present to render the results finite, and to convert them into chirality-violating 
matrix elements. See (C19) for exact formulas. This conversion unfortunately goes against the grain of the spinor helicity technique, which is to make maximum use of chirality conservation. Nevertheless, when external fermion lines are absent, chirality-conservation is not maximally used and the result of the conversion remains to be simple.

Other than the magnetic-moment vertex $S^{\mu}$, which by itself is gauge invariant, everything else is identical to scalar QED. Hence the scattering amplitude is similar to (5.7) and can be written as

$$
A=\int\left[D_{S} \alpha\right] \Delta^{-d / 2}\left\{S^{i n t}(\tilde{q}, p) \exp [-i(M-\tilde{P})]\right\}_{M L}
$$

where $S_{0}^{\text {int }}(q, p)$ receives its contribution from all the modified vertices with the extra factors $(2 m)^{-1}, \frac{1}{2}$, etc., but with the $n_{3}$ external cubic vertices (5.1) removed. As usual, $S^{\text {int }}$ is the sum of $S_{0}^{\text {int }}$ and its contractions. In the special case when internal photon lines are absent (i.e., one-loop $n$-photon amplitudes), the magnetic-moment vertices $S^{\mu}$ have no internal-momentum dependence, then

$$
\begin{aligned}
S^{i n t} & =S_{0}^{i n t}=\frac{1}{2}(-i e)^{m} \operatorname{Tr}\left[\left(\epsilon_{a_{1}} \cdot \sigma \cdot p_{a_{1}}\right)\left(\epsilon_{a_{2}} \cdot \sigma \cdot p_{a_{2}}\right) \cdots\left(\epsilon_{a_{m}} \cdot \sigma \cdot p_{a_{m}}\right)\right] \\
& \equiv \frac{1}{2} \exp \left[-i e \sum_{i, j=1}^{m} \epsilon_{a_{i}} \cdot p_{a_{j}} G_{F}^{a_{i} a_{j}}\right]_{M L}
\end{aligned}
$$

where $a_{1}, a_{2}, \cdots, a_{m}$ are the magnetic-moment vertices around the fermion loop. Using (5.15) and (5.16), the final result in this special case can be written as

$$
A=\frac{1}{2} \int\left[D_{S} \alpha\right] \Delta^{-d / 2} \exp \left[-i(M-\tilde{P})-i e \sum_{a, b=1}^{n_{3}} \epsilon_{a} \cdot p_{b} G_{F}^{a b}\right]_{M L}
$$

which agrees with the results from superstring and first-quantized calculations [22-25].

\subsection{Pure QCD}

There are three terms in a three-gluon vertex. In ordinary covariant gauges, each of these three terms resembles (5.3). In the background gauges discussed in Sec. 4.3, the same Feynman rule holds for internal vertices but not the external ones. In the latter case, one of the three terms, the one with the external gluon dislodged, resembles (5.3), but the other two terms where an internal gluon line is dislodged contain only the momentum of the external gluon. This means that no contraction is needed for these other two terms, and the stringlike reorgainizational techniques of scalar QED can once again be used as follows.

It is helpful to use spin paths to organize the many terms present. Since quarks are absent in pure QCD, there is no reason to use spinor paths; the simpler vector paths would be sufficient. A vector path is a continuous path along the lines of the diagram drawn in such a way that 
1. every gluon line is traversed at most once;

2. a path may be open or closed. If open, it must start and end at external gluon lines;

3. for this purpose a dislodged gluon line is treated like an external line.

A vector covering is a collection of vector paths so that each external and internal gluon line is traversed exactly once.

Spin factors are computed as follows [26]. To the end of each path a vector is assigned. For an external gluon this is its polarization vector; for a dislodged gluon it is the vector shown under the diagrams in Fig. 6. The spin factor $\mathcal{G}_{A}$ for a vector path $\mathrm{A}$ is the dot product of the vectors at the two ends of the path, if the path is open, together with whatever other numerical factors present at the vertices along the path. If the path is closed, then $\mathcal{G}_{A}=g_{\mu}^{\mu}=d$, times other factors in Fig. 6 that the path encountered. The total spin factor $\mathcal{G}$ is once again given by the product of $\mathcal{G}_{A}$ 's in the vector covering.

Except for a factor $(-e)$, the coupling in Fig. 6(b), where the external gluon is dislodged, is identical to the coupling (5.3) in scalar QED. For an external vertex $a$ in which the external gluon line is dislodged, the formulas in Sec. 5.1 apply, viz., $p_{a}$ is replaced by $p_{a}+i e \eta_{a(r)} \epsilon_{a} \partial_{a}$.

There are vector paths where none of the external gluons are dislodged. Some of them, such as those in Figs. 10 and 11, may form a loop passing through $m$ external triple-gluon vertices. We will call these paths $m$-loops. For an $m$-loop, vertices in Figs. 6(c) and 6(d) are relevant. In particular, for something like Fig. 10, the spin factor is

$$
\begin{aligned}
\mathcal{G} & =\prod_{i=1}^{m}\left(-2 e \epsilon_{a_{i}} \cdot p_{a_{i+1}}\right) \\
& =\exp \left[-2 e \sum_{i=1}^{m} \epsilon_{a_{i}} \cdot p_{a_{i+1}}\right]_{M L},
\end{aligned}
$$

where $\left(a_{m+1}=a_{1}, a_{2}, a_{3}, \cdots, a_{m}\right)$ are the ordered external vertices around the $m$-loop.

If the other internal line at one or several vertices $a$ is dislodged instead, Fig. 10 turns into diagrams like Fig. 11. The result in (5.18) is to interchange $\epsilon_{a}$ with $p_{a}$, with a minus sign. The sum of all such contributions is

$$
\mathcal{G}_{m}=\exp \left[2 e \sum_{i=1}^{m}\left(\epsilon_{a_{i}}-p_{a_{i}}\right) \cdot\left(\epsilon_{a_{i+1}}-p_{a_{i+1}}\right)\right]_{M L} .
$$

For a one-loop $n$-gluon amplitude made up of triple gluon vertices, every external gluon line is either dislodged, or is a member of a $m$-loop for some $m$. Thus its amplitude is given by

$$
\begin{aligned}
A=\int\left[D_{S} \alpha\right] \sum_{m \leq n} \exp [ & -i M+i \sum_{a, b=1}^{n}\left(p_{a}+i e \epsilon_{a} \partial_{a}\right) \cdot\left(p_{b}+i e \epsilon_{b} \partial_{b}\right) Z_{a b}(\alpha) \\
& \left.+2 e \sum_{i=1}^{m}\left(\epsilon_{a_{i}}-p_{a_{i}}\right) \cdot\left(\epsilon_{a_{i+1}}-p_{a_{i+1}}\right)\right]_{M L},
\end{aligned}
$$


where the sum in front of the exponential is over all $m$-loops with $m \leq n$, with $\left(a_{1}, \cdots, a_{m}\right)$ arranged in the same cyclic order as the original vertices. This formula agrees with the one obtained from string calculations.

The topology for a general Feynman diagram could be very complicated, in which

case one has to resort back to the general formula (5.15), where $S^{\text {int }}(q, p)$ now includes all the spin factors where the external gluon lines are not dislodged.

\section{Acknowledgement}

This work is supported in part by the Natural Science and Engineering Research Council of Canada and the Québec Department of Education. 


\section{Appendix A. Circuit theory}

Consider an electric circuit with $n$ vertices, each hooked up to an outgoing current $p_{i}(1 \leq i \leq n)$, and $N$ internal lines, each carrying a resistance $\alpha_{r}(1 \leq r \leq N)$ and a current $q_{r}$. If the line $r$ points from vertex $j$ to vertex $i$, then we write $r=(i j)$ and say that the line $r$ and the vertices $i$ and $j$ are mutually adjacent.

Define a $(n \times N)$-dimensional incidence matrix, whose elements $A_{k r}$ are $+1,-1$ respectively if $k=i, j$, and is 0 if $k$ is neither $i$ nor $j$. Current conservation at vertex $i$ is then given by the equation

$$
p_{i}=\sum_{r=1}^{N} A_{i r} q_{r}
$$

and the voltage drop across line $r=(i j)$ is

$$
v_{r}=v_{(i j)}=V_{j}-V_{i}=-\sum_{k=1}^{n} A_{k r} V_{k} .
$$

It is convenient to define a diagonal matrix $\beta$, whose diagonal matrix elements are the conductance $\beta_{r}=1 / \alpha_{r}$ of line $r$. Using Ohm's law

$$
q_{r}=\beta_{r} v_{r}
$$

(A1) and (A2), one gets

$$
p=-\left(A \beta A^{t}\right) V \equiv-Y V
$$

where $p, V$ are $n$-dimensional vectors with components $p_{i}$ and $V_{i}$. From this, one can compute the power consumed by the network to be

$$
P=-V \cdot p=V Y V
$$

Absolute voltage levels in a network are meaningless; voltages can be shifted by a common amount without affecting the physical contents. For example, the invariance of $p$ under this shift can be seen in (A4) from the identity

$$
\sum_{j} Y_{i j}=0
$$

which in turn follows algebraically from the obvious relation $\sum_{i} A_{i r}=0$. Being a symmetric matrix, we must also have $\sum_{i} Y_{i j}=0$, and in (A4) this simply expresses the conservation of the external currents:

$$
\sum_{i=1}^{n} p_{i}=0
$$


Because of (A6), the matrix $Y$ is singular, so (A4) cannot be inverted to obtain $V$ as a function of $p$. This is as it should be because the absolute level of $V$ cannot be determined. In order to invert (A4), a common voltage level must be chosen and fixed. This will be referred to as choosing a level scheme. One simple choice is to ground the last vertex by setting $V_{n}=0$ (the primitive level scheme). The main advantage of this scheme is the easiness to solve for $V$ : (A4) can now be inverted to give

$$
V_{0}=-Y_{0}^{-1} p_{0} \equiv-Z_{0} p_{0},
$$

where the subscript 0 refers to vectors and matrices made up of the first $n-1$ components. Incorporating $V_{n}=0$, we can enlarge this to a $n$-dimensional relation

$$
\begin{aligned}
V & =-Z^{\prime} p, \\
Z^{\prime} & =\left(\begin{array}{cc}
Z_{0} & 0 \\
0 & 0
\end{array}\right),
\end{aligned}
$$

where $Z^{\prime}$ is the impedance matrix. The internal currents and the power are now given in the matrix notation to be

$$
\begin{aligned}
q & =-\beta A^{t} V=\beta A^{t} Z^{\prime} p, \\
P & =-p \cdot V=p Z^{\prime} p .
\end{aligned}
$$

Another useful circuit quantity is the $N \times N$ contraction matrix $H$ used in (2.6). Its matrix element $H_{r s}$, for $r=(i j)$ and $s=(k l)$, is [30]

$$
\begin{aligned}
& H_{r s}=G_{r s}-\beta_{r} \delta_{r s}, \\
& G_{r s}=\left[\beta A^{t} Z^{\prime} A \beta\right]_{r s}=\beta_{r} \beta_{s}\left\{Z_{i k}^{\prime}-Z_{j k}^{\prime}-Z_{i l}^{\prime}+Z_{k l}^{\prime}\right\} .
\end{aligned}
$$

Graph theory can be used to devise rules so that these circuit quantities can be read off directly from a circuit (Feynman) diagram [28-32]. The result is given in eqs. (2.7)-(2.11).

Using (A9) and (A11), $\alpha_{s} G_{r s}$ can be given a simple meaning. It is the current flowing through line $r$ in the presence of a unit external current flowing through the two ends of line $s$ : in at vertex $l$ and out at vertex $k$. It then follows that

$$
\begin{aligned}
& \sum_{s \in \text { loop }} \alpha_{s} G_{r s}=0, \\
& \sum_{s \in \text { loop }} \alpha_{s} H_{r s}=-1,
\end{aligned}
$$

where the sum is taken over the lines $s$ around any closed loop. (A15) is a consequence of (A14) and the first equation of (A13). (A14) is true because the current flowing through $r$ is zero if every vertex around the loop receives both a unit incoming and a unit outgoing current. 
An alternative derivation is to think of $\alpha_{r} \alpha_{s} G_{r s}$ as the voltage drop across line $r$ when the external unit currents are fed through the two ends of line $s$. Since this quantity is symmetrical in $r$ and $s$, we can always interchange $r$ and $s$ in the interpretation. Then $\alpha_{r} \sum_{s \in \text { loop }} \alpha_{s} G_{r s}$ is the total voltage drop around a loop when the unit currents are fed through the two ends of line $r$, and is hence zero.

The impedance matrix is not uniquely defined on account of the $p$-conservation relation (A7). A change

$$
Z_{i j}^{\prime} \rightarrow Z_{i j}=Z_{i j}^{\prime}+\zeta_{i}+\zeta_{j}
$$

shifts the overall level of $V_{i}$ by a common amount $-v=-\sum_{j} \zeta_{j} p_{j}$, but leaves the physical quantities $P, v_{r}, q_{r}$, and $H_{r s}$ invariant. The transformation (A16) is thus a level transformation that changes the level schemes; the invariance means that the matrix $Z^{\prime}$ in the physical quantities (A11)-(A13) may be replaced by the impedance matrix $Z$ in any level scheme. In many ways the choice of a level scheme is analogous to a gauge choice in gauge theories, though the two have absolutely nothing to do with each other.

Physical quantities are defined only on the momentum shell, where constraint (A7) is satisfied. (A9), (A11), and (A12), with $Z^{\prime}$ replaced by a general $Z$, will be used to define $V, q, P$ off-shell as a function of all $p_{i}$. Off-shell extensions are level-scheme (or $Z$ ) dependent, momentum conservation is generally invalid, though Kirchhoff's voltage law, that the total voltage drop along a closed path is zero, still holds. Off-shell quantities are needed in Sec. 5 and Appendix D.

A level scheme that is particularly symmetrical and useful in Sec. 5 is the zero-diagonal level scheme, or simply the diagonal scheme, defined by setting

$$
Z_{i i}=0 \quad \forall i
$$

Its graphical rules can be inferred from (2.8) to be

$$
Z_{i j}=-\frac{1}{2} \sum_{T_{2}^{i j}} \prod^{\ell+1} \alpha, \quad(i \neq j)
$$

where $T_{2}^{i j}$ is the set of all 2-trees with vertices $i$ and $j$ belonging to two separate 1-trees.

We pause now to indicate briefly how (2.14) is obtained from (2.1), and why electric circuit theory becomes relevant in scattering amplitudes [30].

Let $Q=\frac{1}{2} k a k+k b+c$ be a quadratic function of the d-dimensional loop-momentum vectors $k_{a}(1 \leq a \leq \ell)$, and $f_{0}(k)$ be a polynomial function of $k_{a}$. The Euclidean-space Gaussian integral formula

$$
\int\left(\prod_{a=1}^{\ell} d^{d} k_{a}\right) f_{0}(k) \exp (-Q)=\left[\frac{2 \pi}{\operatorname{det}(a)}\right]^{d / 2} f_{0}(-\partial / \partial b) \exp \left(-c+\frac{1}{2} b a^{-1} b\right)
$$

can be used to evaluate (2.1), after (2.13) is used to introduce the Schwinger parameters and a Wick rotation is made. For subsequent manipulation, the first crucial point to note 
is that the exponent on the rhs of (A19) is the value of $-Q$ evaluated at $\partial Q / \partial k_{a}=0$, or at $k=-a^{-1} b$. From (2.1) and (2.13), $Q=-i \sum_{r} \alpha_{r} q_{r}^{2}$. The $N$ internal momenta $q_{r}$ are subject to $n-1$ momentum-conservation constraints (A1), which can be solved to express $q_{r}$ as linear combinations of the external momenta $p_{i}$ and the $\ell=N-n+1$ loop momenta $k_{a}$. The resulting $Q$ is a quadratic function of $k$ as required. Nevertheless, the second crucial observation is that this explicit solution of $q_{r}$ in terms of $k_{a}$ should not be used at this stage. Instead, $\partial Q / \partial k_{a}=0$ should be computed using the Lagrange multiplier method, in which constraints are incorporated into the function $Q^{\prime}=Q-2 i \sum_{i=1}^{n} V_{i}(A q-p)_{i}$ via the Lagrange multipliers $-2 i V_{i}$. Then $\partial Q / \partial k_{a}=0$ can be replaced by $\partial Q^{\prime} / \partial q_{r}=0$, which yields Ohm's law (A3) and (A2). The momentum-conservation equation (A1) then becomes Kirchhoff's current law, and the solution for $q$ is given by (A11). This is how circuit quantities enter into the scattering amplitude formula (2.13), as described in Sec. 2. $\alpha_{r}, q_{r}, p_{i}, P$ become respectively the resistance, the internal current, the external current, and the power of the network. Spacetime points become voltages [30] and translational invariance becomes level invariance.

Suppose the function $f_{0}(k)=S_{0}(q, p)$ is expressed as a function of $q$ and $p$. Eq. (A19) can then be written as

$$
\int\left(\prod_{a=1}^{\ell} d^{d} k_{a}\right) S_{0}(q, p) \exp (-Q)=\left[\frac{2 \pi}{\operatorname{det}(a)}\right]^{d / 2} S(q, p) \exp (i P)
$$

which eventually leads to (2.14). Here $P$ is given by (2.5) and (2.8), $\operatorname{det}(a)$ is essentially $\Delta$ of (2.7). $q$ is given by (2.9) and (A11), and $S=\sum_{k} S_{k}$ is given by the sum of $S_{0}$ and its contractions, computed according to (2.6). In the language of (A19), contraction between a pair of loop momenta $k_{d}$ and $k_{e}$ comes from the differentiation $\left(-\partial / \partial b_{d}\right)\left(-\partial / \partial b_{e}\right) \frac{1}{2} b a^{-1} b=$ $\left(a^{-1}\right)_{d e}$. For more details, please consult Ref. [30].

We turn now to differential circuit identities needed in Sec. 5 and Appendix D. They are most easily derived in the primitive level scheme, though the result is valid in any scheme because only level-independent quantities are involved in the formulas.

Using (A4)-(A13), one gets

$$
\begin{aligned}
& \frac{\partial}{\partial \alpha_{r}} P(\alpha, p)=p \frac{\partial Z^{\prime}}{\partial \alpha_{r}} p=\left(p Z^{\prime} A \beta\right)_{r}\left(\beta A^{t} Z^{\prime} p\right)_{r}=q_{r}^{2} \\
& \frac{\partial}{\partial \alpha_{s}} q_{r}(\alpha, p)=-\beta_{s} \delta_{r s} q_{s}+\left[\beta A^{t} Z^{\prime} A \beta\right]_{r s}\left(\beta A^{t} Z^{\prime} p\right)_{s}=H_{r s} q_{s}
\end{aligned}
$$

These are the two basic identities from which many other identities can be derived.

An important consequence of (A22) is that current conservation leads to a corresponding contraction-matrix conservation. If $\sum_{r} c_{r} q_{r}=0$, then it follows from (A22) that

$$
\sum_{r} c_{r} H_{r s}=0
$$


for every $s$. In particular, if $a$ is an external cubic vertex (Fig. 8), at which momentum conservation reads $p_{a}=q_{a^{\prime \prime}}-q_{a^{\prime}}$, then

$$
H_{a^{\prime} s}=H_{a^{\prime \prime} s}
$$

because $p_{a}$ is $\alpha_{s}$ independent.

The conservation of $H_{r s}$ wrt one of the two indices, while holding the other one fixed [eq. (A23)], together with (A24), show that contraction matrix elements act like bilinear forms of loop momenta. For a $\ell$-loop diagram, there are therefore $\ell(\ell+1) / 2$ independent $H_{r s}$ 's. If necessary, by the polarization trick, they can all be written as linear combinations of diagonal elements, which according to (2.10) are simply derivatives of $\ln (\Delta)$.

The conservation equation (A23) could have been derived from the interpretation of $\alpha_{s} G_{r s}$ as a current, given immediately below (A13). Note that $H_{r s}$ differs from $G_{r s}$ by $-\beta_{r} \delta_{r s}$. The reason why it is $H_{r s}$ but not $G_{r s}$ that is conserved is because there are also external current injection at the two ends of line $s$.

Writing $P=\sum_{r} \alpha_{r} q_{r}^{2}$, using (A22) and (A21), one gets

$$
\sum_{r=1}^{N} \alpha_{r} q_{r} H_{r s}=\sum_{r} v_{r} H_{r s}=0 .
$$

So the voltage drops form an $N$-dimensional null vector of the matrix $H$.

We can also obtain a relation for the derivatives of $H_{r s}$ by differentiating (A22) wrt $\alpha_{t}$ and use the commutativity of the double derivative. Then

$$
\frac{\partial H_{r s}(\alpha)}{\partial \alpha_{t}}=H_{r t}(\alpha) H_{t s}(\alpha) .
$$

So far the relations derived are very general. We shall now derive a set of relations valid only at external cubic vertices $a$ (see Fig. 8).

An important property of an external vertex $a$, which can be seen from the graphical rules (2.7)-(2.11), and (A18), is that $\Delta(\alpha), Z_{i j}$ (in the diagonal level scheme) and $H_{r s}$ depends on $\alpha_{a^{\prime}}$ only through the combination $\alpha_{a^{\prime}}+\alpha_{a^{\prime \prime}}$, provided $a$ is not equal to $i$ or $j$. Let

$$
\partial_{a}=-\sum_{r} A_{a r} \frac{\partial}{\partial \alpha_{r}}=-\frac{\partial}{\partial \alpha_{a^{\prime \prime}}}+\frac{\partial}{\partial \alpha_{a^{\prime}}} .
$$

Then

$$
\partial_{a} \Delta(\alpha)=\partial_{a} H_{r s}=\partial_{a} Z_{i j}=0
$$

provided $i \neq a \neq j$. Similar equations do not exist for internal vertices. This is the reason why the stringlike reorganization discussed in Sec. 5 is valid only for external vertices. 
The first two of these relations also follow from (2.10), (A24), and (A26). The last relation is clearly level-dependent. The fact that it is true in the diagonal level scheme is what makes this scheme particularly useful.

We proceed now to derive other level-dependent relations valid in the diagonal scheme. These relations are useful in Sec. 5. Let

$$
I_{r m}=\beta_{r}\left(Z_{i m}-Z_{j m}\right)
$$

be defined by (2.3) and (A11). It is the current flowing through line $r=(i j)$ when a unit external current flows out of vertex $m$. We will assume $q_{r}$ and $p_{m}$ to flow in the same direction. If not, a minus sign should accompany the appropriate quantities below. Note that this is an off-shell interpretation, so it is level-scheme dependent, and that current conservation is not required or assumed.

Write (A13) in the diagonal scheme as follows,

$$
\left(Z_{i l}-Z_{j l}\right)=\left(Z_{i k}-Z_{j k}\right)-\alpha_{r}\left(\alpha_{s} H_{r s}+\delta_{r s}\right),
$$

where $r=(i j)$ and $s=(k l)$. Let $r=s$ and use (A17). Then

$$
I_{r i}=-\beta_{r} Z_{i j}=\frac{1}{2}\left(\alpha_{r} H_{r r}+1\right)=-I_{r j} .
$$

Next, suppose $s \neq r$. Then (A30) can be written as a recursion formula

$$
I_{r k}=I_{r l}+\alpha_{s} H_{r s} .
$$

Let $s=s_{q}=\left(m i_{q}\right), s_{q-1}=\left(i_{q} i_{q-1}\right), \cdots, s_{1}=\left(i_{2} i\right), r=s_{0}=(i j)$ be a continuous path leading from vertex $j$ to $m$ (see Fig. 12). Using (A32) repeatedly, one gets

$$
I_{r m}=\frac{1}{2}\left(1+\alpha_{r} H_{r r}\right)+\sum_{p=1}^{q} \alpha_{s_{p}} H_{r s_{p}} .
$$

Suppose $a$ is an external cubic vertex (see Fig. 8) and suppose $i \neq j$. Using (A28) and (A33), one gets

$$
\partial_{a} I_{r m}=-\rho_{a r m} H_{r a^{\prime}},
$$

where

$$
\rho_{\text {arm }}= \begin{cases}0, & \text { if } a \neq j, i, m ; \\ -\frac{1}{2}, & \text { if } a=j \neq m \text { or } a=i \neq m ; \\ \frac{1}{2}, & \text { if } a=i=m \text { or } a=m=j ; \\ 1, & \text { if } a=m \neq i, j .\end{cases}
$$

Note that $a=j=m$ indicates the presence of a closed loop. The relations for $a=m$ will be particularly important. Let

$$
\rho_{a r} \equiv \rho_{a r a}= \begin{cases}1, & \text { if } A_{a r}=0 \\ \frac{1}{2}, & \text { if } A_{a r} \neq 0 .\end{cases}
$$


Then

$$
\partial_{a} I_{r a}=-\rho_{a r} H_{a^{\prime} r} .
$$

This equation will be used to replace contraction of external cubic vertices by differentiation. See Sec. 5 and Appendix D.

Next, assume $a=i \neq m$ to be an external cubic vertex and use (A29) to compute

$$
\partial_{i} I_{r m}=\beta_{r}\left(I_{r m}+\partial_{i} Z_{i m}\right)
$$

Using (A35), this gives

$$
\partial_{i} Z_{i m}=\frac{1}{2} \alpha_{r} H_{r r}-I_{r m}
$$

which can be written in a form independent of the label and the postition of $i$, as long as $a \neq m$ :

$$
\partial_{a} Z_{a m}=\frac{1}{2} \alpha_{a^{\prime \prime}} H_{a^{\prime \prime} a^{\prime \prime}}-I_{a^{\prime \prime} m} .
$$

Similarly, using (A29) and (A35), one gets

$$
\partial_{j} Z_{j m}=-\frac{1}{2} \alpha_{r} H_{r r}-I_{r m}
$$

which in a postition independent form reads

$$
\partial_{a} Z_{a m}=-\frac{1}{2} \alpha_{a^{\prime}} H_{a^{\prime} a^{\prime}}-I_{a^{\prime} m}
$$

Adding (A39) and (A40), one deduces that as long as $a \neq m$,

$$
-2 \partial_{a} Z_{a m}=\frac{1}{2}\left(\alpha_{a^{\prime}}-\alpha_{a^{\prime \prime}}\right) H_{a^{\prime} a^{\prime}}+\left(I_{a^{\prime} m}+I_{a^{\prime \prime} m}\right)
$$

It is easy to verify that this relation holds for $a=m$ as well. Note however that relations (A39) and (A40) individually are not valid at $a=m$.

Multiply (A41) by $p_{m}$ and sum over $m$. Using the momentum-conservation equation (A7), one gets

$$
-2 \sum_{m=1}^{n} \partial_{a} Z_{a m} p_{m}=q_{a^{\prime}}+q_{a^{\prime \prime}}
$$

Using the explicit form for $I_{r m}$, it is easy to obtain from (A41) that if $b=m \neq a$ is also an external cubic vertex, then

$$
\partial_{a} \partial_{b} Z_{a b}=H_{a^{\prime} b^{\prime}}
$$

It should be emphasized once again that these level-dependent relations are vald only in the diagonal scheme. 


\section{Appendix B: Color factors}

Let $T^{a}$ be the $U\left(N_{c}\right)$ generators in the fundamental representation normalized according to (3.3). The completeness relation dual to (3.3) is

$$
\sum_{a=0}^{N_{c}^{2}-1}\left(T^{a}\right)_{i j}\left(T^{a}\right)_{k l}=\delta_{i l} \delta_{k j} .
$$

This can be used to sum up intermediate color indices as follows. Let $X, Y, U, V$ each be a product of these generator matrices. Then (B1) implies

$$
\sum_{a=0}^{N_{c}^{2}-1}\left(X T^{a} Y\right)_{i j}\left(U T^{a} V\right)_{k l}=(X V)_{i l}(U Y)_{k j}
$$

which is the basis for most of the rules for a color path. In particular, rule 3 .

When a three-gluon vertex or a ghost-gluon vertex with colors $a, b, c$ is encountered, the color factor for the vertex is the Lie-algebra structure constant

$$
f^{a b c}=-i\{\operatorname{Tr}(a b c)-\operatorname{Tr}(a c b)\}
$$

where $a, b, c$ on the rhs stand for $T^{a}, T^{b}, T^{c}$, respectively. When a four-gluon vertex is encountered, the color factor is

$$
f^{a b c d} \equiv \sum_{e} f^{a b e} f^{e c d}=(-i)^{2}\{\operatorname{Tr}(a b c d)-\operatorname{Tr}(b a c d)-\operatorname{Tr}(a b d c)+\operatorname{Tr}(b a d c)\}
$$

In particular, this shows the decoupling between the $U(1)$ and the $S U\left(N_{c}\right)$ in $U\left(N_{c}\right)$ :

$$
f^{0 b c}=f^{0 b c d}=0 .
$$

Eqs. (B3) and (B4) are the basis of rule 5 stated in Sec 3.2 for a color path. The predetermined order stated in the rule is determined by which of the terms in (B3) and (B4) are chosen.

Modifications are necessary for $S U\left(N_{c}\right)$ theories because it has only $N_{c}^{2}-1$ generators. The completeness relation is now replaced by

$$
\sum_{a=1}^{N_{c}^{2}-1}\left(T^{a}\right)_{i j}\left(T^{a}\right)_{k l}=\delta_{i l} \delta_{k j}-\frac{1}{N_{c}} \delta_{i j} \delta_{k l} .
$$

An exception occurs if somewhere in the connected gluon network a three-gluon, a fourgluon, or a ghost-gluon vertex occurs. Then because of (B5), we may effectively sum over all $N_{c}^{2}$ color vertices again, so the correction term on the rhs of (B6) is no longer necessary. 
As a result, color paths and color factors of $S U\left(N_{c}\right)$ are identical to those of $U\left(N_{c}\right)$, except with the following additions corresponding to the last term of (B6). Two quark lines, or two protions of the same quark line, may be linked by a single, or more than one gluon lines. In the latter case, everything is the same as $U\left(N_{c}\right)$. In the former case, a new option is available when a quark-gluon vertex is encountered while travelling along a quark line. If this new option is taken, the path will continue along the same quark line passed the vertex. At the same time, the path along the other quark line, passing through the other end of the single internal gluon line connecting the two, must also adopt the new option of going straight forward. The color factor with this new option carries an extra $\left(-N_{c}^{-1}\right)$ factor but would otherwise be the same as in the $U\left(N_{c}\right)$ case.

In a color covering where new options are taken, the single internal gluon lines in question will never be traversed by any color path, whereas all the other gluon lines would be traversed twice as before.

For example, the additional color coverings for Fig. 1 in the case of $S U\left(N_{c}\right)$ are $\left\{v_{1}=\right.$ $\left.\left(p_{2} q_{2} q_{3} p_{3}\right), v_{2}=\left(p_{4} q_{2} q_{1} p_{1}\right)\right\},\left\{v_{1}=\left(p_{2} q_{1} q_{4} p_{3}\right), v_{2}=\left(p_{4} q_{3} q_{4} p_{1}\right)\right\}$, and $\left\{v_{1}=\left(p_{2} q_{1} p_{1}\right), v_{2}=\right.$ $\left.\left(p_{4} q_{3} p_{3}\right)\right\}$. The corresponding color factors are $-N_{c}^{-1}(\mathbf{1})_{i j^{\prime}}(\mathbf{1})_{i^{\prime} j},-N_{c}^{-1}(\mathbf{1})_{i j^{\prime}}(\mathbf{1})_{i^{\prime} j}$, and $+N_{c}^{-2}(\mathbf{1})_{i j}(\mathbf{1})_{i^{\prime} j^{\prime}}$.

\section{Appendix C. Spinor helicity technique}

The massless Dirac spinor in the chiral representation where $\gamma_{5}$ is diagonal can be taken to be

$$
\begin{aligned}
& \left.u_{+}(p)\right|_{m=0} \equiv|p+\rangle=\sqrt{2 p^{0}}\left(\begin{array}{c}
\chi_{+}(\vec{p}) \\
0
\end{array}\right), \\
& \left.u_{-}(p)\right|_{m=0} \equiv|p-\rangle=-\sqrt{2 p^{0}}\left(\begin{array}{c}
0 \\
\chi_{-}(\vec{p})
\end{array}\right) \text {, } \\
& \left.\bar{u}_{+}(p)\right|_{m=0} \equiv\langle p+|=-\sqrt{2 p^{0}}\left(\begin{array}{ll}
0 & \left.\chi_{+}^{*}(\vec{p})\right)
\end{array}\right) \text {, } \\
& \left.\bar{u}_{-}(p)\right|_{m=0} \equiv\langle p-|=\sqrt{2 p^{0}}\left(\chi_{-}^{*}(\vec{p}) \quad 0\right) \text {, }
\end{aligned}
$$

where the two-component helicity eigenstate $\chi_{\lambda}(\vec{p})$ satisfies

$$
\begin{aligned}
\vec{\sigma} \cdot \vec{p} \chi_{\lambda}(\vec{p}) & =\lambda|\vec{p}| \chi_{\lambda}(\vec{k}), \\
\chi_{\lambda^{\prime}}^{*}(\vec{p}) \chi_{\lambda}(\vec{p}) & =\delta_{\lambda^{\prime} \lambda},
\end{aligned}
$$

and $p$ is in the forward light cone. The normalization adopted in (C1) gives

$$
\left\langle p \lambda\left|\gamma^{\mu}\right| p \lambda\right\rangle=2 p^{\mu}
$$

In particular, $\xi p$ is in the forward light cone if $p$ is and if $\xi>0$, then

$$
\begin{aligned}
|(\xi p) \lambda\rangle & =\sqrt{\xi}|p \lambda\rangle, \\
\langle(\xi p) \lambda| & =\sqrt{\xi}\langle p \lambda|(C 4)
\end{aligned}
$$


Chirality conservation implies

$$
\langle p \pm \mid q \pm\rangle=0
$$

The non-zero matrix elements are

$$
\begin{aligned}
& \langle p q\rangle=\langle p-\mid q+\rangle=2 \sqrt{p^{0} q^{0}} \chi_{-}^{*}(\vec{p}) \chi_{+}(\vec{q}) \\
& {[p q]=\langle p+\mid q-\rangle=2 \sqrt{p^{0} q^{0}} \chi_{+}^{*}(\vec{p}) \chi_{-}(\vec{q}) .}
\end{aligned}
$$

Since $\sigma_{2} \vec{\sigma} \sigma_{2}=-\vec{\sigma}^{*}$, it is convenient to choose the phase of the helicity eigenstates to satisfy

$$
\sigma_{2} \chi_{\lambda}(\vec{p})=i \lambda \chi_{-\lambda}^{*}(\vec{p}) .
$$

It also determines the phase under charge conjugation. This then implies

$$
\chi_{ \pm}^{*}(\vec{p}) \chi_{\mp}(\vec{q})=\chi_{ \pm}^{*}(\vec{p}) \sigma_{2} \sigma_{2} \chi_{\mp}(\vec{q})=-\chi_{\mp}^{*}(\vec{q}) \chi_{ \pm}(\vec{p})
$$

or equivalently,

$$
\langle p q\rangle=-\langle q p\rangle, \quad[p q]=-[q p]=\operatorname{sign}(p \cdot q)\langle q p\rangle^{*} .
$$

Note that both $p$ and $q$ are assumed to be in the forward light cone so $\partial \cdot q \geq 0$.

This phase also gives rise to the relations

$$
\begin{aligned}
\left\langle p \pm\left|\gamma_{\mu_{1}} \cdots \gamma_{\mu_{2 n+1}}\right| q \pm\right\rangle & =\left\langle q \mp\left|\gamma_{\mu_{2 n+1}} \cdots \gamma_{\mu_{1}}\right| p \mp\right\rangle \\
\left\langle p \pm\left|\gamma_{\mu_{1}} \cdots \gamma_{\mu_{2 n}}\right| q \mp\right\rangle & =-\left\langle q \pm\left|\gamma_{\mu_{2 n}} \cdots \gamma_{\mu_{1}}\right| p \mp\right\rangle
\end{aligned}
$$

Using (C3) and

$$
\gamma p=|p+\rangle\langle p+|+| p-\rangle\langle p-|,
$$

it is easy to see that

$$
\langle p+|\gamma k| q+\rangle=[p k]\langle k q\rangle, \quad\langle p-|\gamma k| q-\rangle=\langle p k\rangle[k q] .
$$

In particular, using (C3), one gets

$$
\langle q p\rangle[p q]=2(p \cdot q)
$$

Thus up to phases, both $\langle p q\rangle$ and $[p q]$ are equal to the square root of $2 p \cdot q$. This equation also dictates the sign factor $\operatorname{sign}(p \cdot q)$ of $(\mathrm{C} 9)$ when $p \cdot q$ takes on a negative value.

Fierz identities give the relations

$$
\begin{aligned}
\langle A D\rangle\langle C B\rangle+\langle A C\rangle\langle B D\rangle & =\langle A B\rangle\langle C D\rangle, \\
\left\langle A+\left|\gamma_{\mu}\right| B+\right\rangle\left\langle C-\left|\gamma^{\mu}\right| D-\right\rangle & =2[A D]\langle C B\rangle, \\
\left\langle A+\left|\gamma_{\mu}\right| B+\right\rangle\left\langle C+\left|\gamma^{\mu}\right| D+\right\rangle & =2[A C]\langle D B\rangle .
\end{aligned}
$$


The spin factors for fermions discussed in Secs. 4.2 and 4.3 are based on eqs. (C13), (C16), and (C17).

To order $(m / p)$, the Dirac wave functions for small but finite mass $m$ are

$$
\begin{aligned}
& u_{ \pm}(p)=|p \pm\rangle+\frac{m}{2 p^{0}}|\tilde{p} \mp\rangle, \\
& \bar{u}_{ \pm}(p)=\langle p \pm|+\frac{m}{2 p^{0}}\langle\tilde{p} \mp|,
\end{aligned}
$$

where $\tilde{p}=\left(p^{0},-\vec{p}\right)$ when $p=\left(p^{0}, \vec{p}\right)$. If $\Gamma$ is a product of an even number of $\gamma$-matrices, then $\langle p \pm|\Gamma| q \pm\rangle=0$, but the limit as $m \rightarrow 0$ of the following exists and can be computed from $(\mathrm{C} 18)$ :

$$
\lim _{m \rightarrow 0}\left\{m^{-1} \bar{u}_{ \pm}(p) \Gamma u_{ \pm}(q)\right\}=\frac{1}{2 p^{0}}\langle\tilde{p} \mp|\Gamma| q \pm\rangle+\frac{1}{2 q^{0}}\langle p \pm|\Gamma| \tilde{q} \mp\rangle .
$$

These relations can be used in Sec. 5.2 to calculate the matrix elements of the transformed vertices.

The photon/gluon polarization vector with helicity \pm 1 can be chosen to be

$$
\epsilon_{ \pm}^{\mu}(p, k)= \pm \frac{\left\langle p \pm\left|\gamma^{\mu}\right| k \pm\right\rangle}{\sqrt{2}\langle k \mp \mid p \pm\rangle}
$$

where the reference momentum $k$ in $(\mathrm{C} 18)$ is massless but otherwise arbitrary. The choice of different $k$ corresponds to the choice of a different gauge, and these different choices are related by

$$
\epsilon_{+}^{\mu}(p, k) \rightarrow \epsilon_{+}^{\mu}\left(p, k^{\prime}\right)-\sqrt{2} \frac{\left\langle k k^{\prime}\right\rangle}{\langle k p\rangle\left\langle k^{\prime} p\right\rangle} p^{\mu} .
$$

These polarization vectors satisfy the following identities:

$$
\begin{aligned}
\epsilon_{ \pm}^{\mu}(p, k) & =\left(\epsilon_{\mp}^{\mu}(p, k)\right)^{*}, \\
\epsilon_{ \pm}(p, k) \cdot p & =\epsilon_{ \pm}(p, k) \cdot k=0, \\
\epsilon_{ \pm}(p, k) \cdot \epsilon_{ \pm}\left(p, k^{\prime}\right) & =0, \\
\epsilon_{ \pm}(p, k) \cdot \epsilon_{\mp}\left(p, k^{\prime}\right) & =-1, \\
\epsilon_{ \pm}(p, k) \cdot \epsilon_{ \pm}\left(p^{\prime}, k\right) & =0, \\
\epsilon_{ \pm}(p, k) \cdot \epsilon_{\mp}\left(k, k^{\prime}\right) & =0, \\
\epsilon_{+}^{\mu}(p, k) \epsilon_{-}^{\nu}(p, k)+\epsilon_{-}^{\mu}(p, k) \epsilon_{+}^{\nu}(p, k) & =-g^{\mu \nu}+\frac{p^{\mu} k^{\nu}+p^{\nu} k^{\mu}}{p \cdot k}, \\
\gamma \cdot \epsilon_{ \pm}(p, k) & = \pm \frac{\sqrt{2}}{\langle k \mp \mid p \pm\rangle}(|p \mp\rangle\langle k \mp|+| k \pm\rangle\langle p \pm|) .
\end{aligned}
$$

The spin factors for photons/gluons given in Secs. 4.2 and 4.3 are direct results of these equations. 
See the last paper in Ref. [16] for an excellent review of the spinor helicity technique and its application to tree amplitudes.

\section{Appendix D. Gauge invariance}

We discuss in this appendix local gauge invariance in the Schwinger representation, and from there the stringlike formula (5.7) for scalar QED.

Let us first review how local gauge invariance is usually proved in the momentum space. Consider the three diagrams in Fig. 13. Each of them is meant to be a part of a larger, but otherwise common, diagram. Let $\epsilon_{a}$ be the polarization vector of the external photon at vertex $a$. The vertex $j$ may be internal or external. A gauge transformation produces the change

$$
\delta \epsilon_{a}=\lambda_{a} p_{a}
$$

for some infinitesimal gauge parameter $\lambda_{a}$, which in turn produces the following changes in Figs. 13(a), (b), and (c):

$$
\begin{aligned}
\delta_{a} & =\lambda_{a} e^{2} p_{a} \cdot\left[\left(q+p_{a}\right)+q\right] \frac{1}{-\left(q+p_{a}\right)^{2}}\left[\left(q^{\prime}-p_{j}\right)+q^{\prime}\right]^{\nu} \\
& =\lambda_{a} e^{2}\left[\left(q+p_{a}\right)^{2}-q^{2}\right] \frac{1}{-\left(q+p_{a}\right)^{2}}\left[2 q^{\prime}-p_{j}\right]^{\nu} \\
& \rightarrow-\lambda_{a} e^{2}\left[2 q^{\prime}-p_{j}\right]^{\nu}, \\
\delta_{b} & =\lambda_{a} e^{2} p_{a} \cdot\left[\left(q^{\prime}-p_{a}\right)+q^{\prime}\right] \frac{1}{-\left(q^{\prime}-p_{a}\right)^{2}}\left[\left(q+p_{j}\right)+q\right]^{\nu} \\
& =\lambda_{a} e^{2}\left[q^{\prime 2}-\left(q^{\prime}-p_{a}\right)^{2}\right] \frac{1}{-\left(q^{\prime}-p_{a}\right)^{2}}\left[2 q+p_{j}\right]^{\nu} \\
& \rightarrow \lambda_{a} e^{2}\left[2 q+p_{j}\right]^{\nu}, \\
\delta_{c} & =2 \lambda_{a} e^{2} p_{a}^{\nu} .
\end{aligned}
$$

The arrows single out the term that cancels the propagator. The remaining term will cancel another propagator (not shown) on the other side of the vertex. Thus the total local change is

$$
\delta_{a}+\delta_{b}+\delta_{c} \rightarrow-e^{2} \lambda_{a}\left[\left(2 q^{\prime}-p_{j}\right)-\left(2 q+p_{j}\right)-2 p_{a}\right]^{\nu}=0
$$

which shows local gauge invariance at an external vertex.

In short, the proof depends on the cancellation of an adjacent propagator from the divergence of the vertex, thereby reducing both Figs. 13(a) and 13(b) to the seagull form $13(\mathrm{c})$, and the coefficients of these three identical diagrams add up to zero.

In spite of its similarity to (2.1), this proof of local gauge invariance will not work for (2.14) because of the presence of the additional terms $S_{k}$, and because the propagator $T$ in (2.15) can no longer be canceled by the divergence of the vertex factor. Instead, we shall have to adopt a new technique in which Figs. 13(a) and 13(b) are turned into the seagull form Fig. 13(c) by shrinking the relevant propagators, rather than by canceling them. 
The success of this depends crucially on the two differential circuit identities (A21) and (A22). Under the gauge change (D1), the change of the vertex $C_{a}=e \epsilon_{a} \cdot\left(q_{a^{\prime}}+q_{a^{\prime \prime}}\right)$ can be obtained from (A21) and the conservation equation

$$
p_{a}=q_{a^{\prime \prime}}-q_{a^{\prime}}
$$

to be

$$
\delta C_{a}=\lambda_{a} e p_{a} \cdot\left(q_{a^{\prime}}+q_{a^{\prime \prime}}\right)=-\lambda_{a} e \partial_{a} P,
$$

where $\partial_{a}$ is defined in (5.10). The gauge change of the contraction of $C_{a}$ with any internal momentum $q_{r}$ is

$$
\delta\left(C_{a} \sqcup q_{r}\right)=\lambda_{a}(-i e / 2)\left(H_{a^{\prime} r}+H_{a^{\prime \prime} r}\right) p_{a}=-i e \lambda_{a} H_{a^{\prime} r} p_{a}
$$

when (A24) is used, and using (A22) this is equal to

$$
\lambda_{a}\left(i e \partial_{a}\right) q_{r}=i e \lambda_{a}\left(H_{a^{\prime} r} q_{a^{\prime}}-H_{a^{\prime \prime} r} q_{a^{\prime \prime}}\right)=-i e \lambda_{a} H_{a^{\prime} r} p_{a} .
$$

This means that the momenta in $C_{a}$ and their contractions can both be obtained from the operator $i e \partial_{a}$.

Consequently, if $C_{a}$ is factored out of the numerator factor $S_{0}$ in (2.1),

$$
S_{0}(q, p)=C_{a} S_{0}^{\prime}(q, p),
$$

the gauge change of the amplitude

$$
A=\int\left[D_{S} \alpha\right] \Delta^{-d / 2} S(q, p) \exp [-i\{M-P\}]
$$

under (D1) is simply

$$
\delta A=\int\left[D_{S} \alpha\right]\left(i e \lambda_{a} \partial_{a}\right)\left\{\Delta^{-d / 2} S^{\prime}(q, p) \exp [-i(M-P)]\right\},
$$

where $S^{\prime}=\sum_{k} S_{k}^{\prime}$ is the sum of $S_{0}^{\prime}$ and all its contractions.

Instead of having the propagators cancelled, as in (D2), local gauge invariance under (D1) is now accomplished by the operator $\left(i e \partial_{a}\right)$, which upon integration, produce two terms of opposite signs respectively with $\alpha_{a}^{\prime}$ and $\alpha_{a}^{\prime \prime}$ zero. Since the $\alpha$ 's are resistances, setting them zero is equivalent to short-circuiting that branch, which effectively turns Figs. 13(a) and 13(b) into the seagull form of Fig. 13(c). The rest of the proof of local gauge invariance is similar to (D3).

Under a reparametrization transformation of the proper time $\tau$, a function $f(\tau)$ is changed by an amount $(\delta \tau)(/ p f(\tau) / \partial \tau)$. Taking the Schwinger parameters as proper-time parameters, eq. (D10) can be interpreted as a special kind of proper-time transformation 
of the integrand of (D9), with the vertex a removed. In that sense a gauge transformation can be regarded as a proper-time reparametrization. This is tentalizing because in string theories gauge invariance follows from worldsheet reparametrization and conformal invariances. In the infinite tension limit, where string theory becomes a gauge theory, the worldsheet variable $\sigma$ disappears leaving behind only the proper time $\tau$. Conformal invariance also vanishes so only proper-time reparametrization is left, nevertheless gauge invariance still holds. It is therefore tempting to relate gauge invariance with proper-time reparametrization invariance. Indeed such a relation exists at least in the form of eq. (D10).

We can now repeat the same argument and compute the change of the quantity inside the curly bracket of (D10) when another external photon $b$ undergoes a gauge change, and so on down the line to obtain the total change to be

$$
\begin{aligned}
\delta A & =\int\left[D_{S} \alpha\right]\left(i e \prod_{a=1}^{n_{3}} \lambda_{a} \partial_{a}\right)\left\{\Delta^{-d / 2} S^{i n t}(q, p) \exp [-i(M-P)]\right\} \\
& \equiv \int\left[D_{S} \alpha\right] \exp \left[i e \sum_{a=1}^{n_{3}} \lambda_{a} \partial_{a}\right]_{M L}\left\{\Delta^{-d / 2} S^{i n t}(q, p) \exp [-i(M-P)]\right\}
\end{aligned}
$$

where $S^{i n t}(q, p)$ is the same internal-vertex numerator function used in (5.7). The subscript ML for the exponential indicates that only the term multilinear in all the $\partial_{a}$ should be kept when that exponential is expanded into power series.

Eq. (D11) has the advantage that contractions with, and among, the external vertices $C_{a}$ are automatically carried out by the operators $i e \lambda_{a} \partial_{a}$, which according to (D5) are needed to produce the internal momenta at the vertices $a$ to begin with. Now $\delta A$ is obtained from $A$ by having $\epsilon_{a}$ replaced by $\lambda_{a} p_{a}$. Conversely, one might think that $A$ can be obtained from $\delta A$ by replacing $\lambda_{a} p_{a}$ with $\epsilon_{a}$, or by replacing $\left[i e \lambda_{a} \partial_{a}\right]$ with $\left[i e \partial_{a} \epsilon_{a} \cdot\left(\partial / \partial p_{a}\right)\right]$. In that case, one gets

$$
\begin{aligned}
A & =\int\left[D_{S} \alpha\right] \exp \left[i e \sum_{a=1}^{n_{3}} \epsilon_{a} \cdot D_{a}\right]_{M L}\left\{\Delta^{-d / 2} S^{i n t}(q, p) \exp [-i(M-P)]\right\} \\
D_{a}^{\mu} & \equiv \partial_{a} \frac{\partial}{\partial p_{a \mu}} .
\end{aligned}
$$

By carrying out the momentum translation implied by the first exponential factor, one gets (5.7) (except for the $\eta_{a s}$ factor in (5.11)).

The argument given above, though simple and intuitive, is actually full of flaws. For one thing (D13) is meaningful only when we know how to extend the functions it operates on off-shell (see Appendix A), but no specification has been given in (D12) as to what level scheme to be used for such an off-shell extension. Another point is that while it is completely correct to obtain $\delta A$ from $A$ by the substitution $\epsilon_{a} \rightarrow \lambda_{a} p_{a}$, the inverse substitution to get from $\delta A$ to $A$ is full of uncertainties because one would not know which $p_{a}$ 's in $\delta A$ are to be so replaced. The argument leading to (D12) was presented mainly to show the closeness between the stringlike formula and the local gauge invariance considerations. Beyond that it should not be taken seriously. 
The correct derivation makes use of (A37), (A42), and (A43). If $a, b$ are external cubic vertices, and there are $n_{3}$ of those altogether, then (A42) and (A43) show that the external cubic vertices $\prod_{a=1}^{n_{3}} C_{a}=e \prod_{a=1}^{n_{3}} \epsilon_{a} \cdot\left(q_{a^{\prime}}+q_{a^{\prime \prime}}\right)$, as well as their mutual contractions $C_{a} \sqcup C_{b}=-2 i e^{2} \epsilon_{a} \cdot \epsilon_{b} H_{a^{\prime} b^{\prime}}$, can all be obtained from $\exp (i P)=\exp \left(i \sum_{i, j} p_{i} \cdot p_{j} Z_{i j}\right)$ by shifting $p_{a}$ to $p_{a}+i e \epsilon_{a} \partial_{a}$, and taking the terms multilinear (ML) in the $\epsilon_{a}$ 's. This proves (5.7) when $S_{0}^{\text {int }}=1$. When $S_{0}^{\text {int }}(q, p) \neq 1$, contractions of pairs of $q$ 's within $S_{0}^{\text {int }}$, as well as contractions of those in $S_{0}^{\text {int }}$ with those in $C_{a}$ are necessary. The former turns $S_{0}^{\text {int }}$ into $S^{i n t}(q, p)$, and the latter, $C_{a} \sqcup q_{r}=-i e \epsilon_{a} H_{a^{\prime} r}$, according to (A37) can be accomplished by shifting $p_{a}$ within $q_{r}$ to $p_{a}+i e \rho_{a r}^{-1} \epsilon_{a} \partial_{a}$, as stated in (5.7) and (5.9).

So far we have considered gauge invariance for external photon lines. The proof of local gauge invariance for internal photon lines is similar but more complicated. The main difference is that momentum conservation and (A23) have to be used more extensively. Here is how it goes.

The vertex $i$ in Fig. 14 is meant to be the same as the vertex $a$ in Figs. 13a, except that it is now internal. Being internal, the photon line with momenta $p_{i}$ must connect to another charged line at a vertex $k$, as shown. Factor out the explicit factors connecting vertices $i$ and $k$, we can write the numerator function $S_{0}$ of (2.1) in the Feynman gauge as

$$
S_{0}=e^{2}\left[\left(q_{i^{\prime \prime}}+q_{i^{\prime}}\right) \cdot\left(q_{k^{\prime \prime}}+q_{k^{\prime}}\right)\right] S_{0}^{\prime} .
$$

Using (5.5), a gauge change of the photon joining $i$ and $k$ would lead to a change

$$
\delta S_{0}=(\delta \xi)\left[p_{i} \cdot\left(q_{i^{\prime \prime}}+q_{i^{\prime}}\right)\right]\left[p_{i} \cdot\left(q_{k^{\prime \prime}}+q_{k^{\prime}}\right)\right] p_{i}^{-2} S_{0}^{\prime} .
$$

Strictly speaking, the denominator $p_{i}^{-2}$ above should not appear in $\delta S_{0}$. When we go over to the parametric representation (2.14), this factor can be replaced by a factor $i \alpha_{i}$, so we can effectively ignore this factor from now on.

Defining

$$
\partial_{i}=\partial / \partial \alpha_{i^{\prime}}-\partial / \partial \alpha_{i^{\prime \prime}}, \quad \partial_{k}=\partial / \partial \alpha_{k^{\prime}}-\partial / \partial \alpha_{k^{\prime \prime}},
$$

then the claim is that the change of $A$ in (2.14) is given by

$$
\delta A=(\delta \xi) \int\left[D_{S} \alpha\right]\left(i e \partial_{i}\right)\left(-i e \partial_{k}\right)\left\{i \alpha_{i} \Delta^{-d / 2}(\alpha) S^{\prime}(q, p) \exp [-i(M-P)]\right\},
$$

where $S^{\prime}(q, p)$ is the sum of $S_{0}^{\prime}$ and its contractions $S_{k}^{\prime}$. The proof follows.

Using (A21) and momentum conservation, it follows like before that

$$
\partial_{i} P=-p_{i} \cdot\left(q_{i^{\prime \prime}}+q_{i^{\prime}}\right), \quad \partial_{k} P=p_{i} \cdot\left(q_{k^{\prime \prime}}+q_{k^{\prime}}\right) .
$$

To show that these differential operators $\partial_{i}$ and $\partial_{k}$ also generates contractions correctly, let us take an internal momentum $q_{r}$ and compute its contraction with $p_{i} \cdot\left(q_{i^{\prime \prime}}+q_{i^{\prime}}\right)$ :

$$
p_{i} \cdot\left(q_{i^{\prime \prime}}+q_{i^{\prime}}\right) \sqcup q_{r}=-(i / 2)\left[H_{i r}\left(q_{i^{\prime \prime}}+q_{i^{\prime}}\right)+\left(H_{i^{\prime \prime} r}+H_{i^{\prime} r}\right) p_{i}\right] .
$$


On the other hand, using (A22),

$$
\left(i \partial_{i}\right) q_{r}=i\left[H_{i^{\prime} r} q_{i^{\prime}}-H_{i^{\prime \prime} r} q_{i^{\prime \prime}}\right]=(i / 2)\left[\left(H_{i^{\prime} r}-H_{i^{\prime \prime} r}\right)\left(q_{i^{\prime}}+q_{i^{\prime \prime}}\right)+\left(H_{i^{\prime} r}+H_{i^{\prime \prime} r}\right)\left(q_{i^{\prime}}-q_{i^{\prime \prime}}\right)\right] .
$$

Using momentum conservation and contraction-function conservation (A23), eqs. (D20) and (D19) are seen to be identical. So $\left(i \partial_{i}\right)$ does produce the correct contraction with any internal momentum $q_{r}$, and similarly the same is true for $\left(-i \partial_{k}\right)$.

The remaining item to consider is the effect of $\left(i \partial_{i}\right)$ and $\left(-i \partial_{k}\right)$ on $\Delta^{-d / 2}$ and where that comes from. According to (2.10),

$$
\left(i \partial_{i}\right) \Delta^{-d / 2}=(i d / 2) \Delta^{-d / 2}\left(H_{i^{\prime} i^{\prime}}-H_{i^{\prime \prime} i^{\prime \prime}}\right) .
$$

The factor $(i d / 2) \Delta^{-d / 2}\left(H_{i^{\prime} i^{\prime}}-H_{i^{\prime \prime} i^{\prime \prime}}\right)$ is what one would get from the contraction within the factor $-p_{i} \cdot\left(q_{i^{\prime \prime}}+q_{i^{\prime}}\right)$,

$$
-p_{i} \sqcup\left(q_{i^{\prime \prime}}+q_{i^{\prime}}\right)=(i / 2) g_{\mu}^{\mu}\left(H_{i i^{\prime}}+H_{i i^{\prime \prime}}\right),
$$

when $d=g_{\mu}^{\mu}$ and (A23) are used. Other contractions can also obtained by differentiations. Thus eq. (D17) is valid. The rest of the proof is very similar to the situation in momentum space.

Once again eq. (D17) supports the interpretation of gauge invariance to be a propertime reparametrization invariance.

\section{Appendix E. Gordon Identity}

The off-shell Gordon identity used in Sec. 5.2 was proven in Ref. [27]. An alternative, diagramatic, proof will be given in this appendix.

The central identity to use is

$$
e\left[\gamma A\left(m+\gamma q^{\prime}\right)-\left(m-\gamma q^{\prime \prime}\right) \gamma A\right]=C^{\mu} A_{\mu}+S^{\mu} A_{\mu},
$$

where

$$
\begin{aligned}
C^{\mu} & =e\left(q^{\prime}+q^{\prime \prime}\right)^{\mu}, \\
S^{\mu} & =-i e \sigma^{\mu \nu}\left(q^{\prime}-q^{\prime \prime}\right)_{\nu} .
\end{aligned}
$$

Apply this to Fig. 13a, now considered as a part of a spinor QED diagram, with $q^{\prime \prime} \equiv$ $q+p_{a}=q^{\prime}-p_{j}$ and spinor vertices $F^{\mu} A_{\mu}=e \gamma^{\mu} A_{\mu}$ :

$e^{2} \gamma A_{a}\left[m-\gamma q^{\prime \prime}\right]^{-1} \gamma A_{j}\left[m-\gamma q^{\prime}\right]^{-1}=e \gamma A_{a}\left\{e \gamma A_{j}+\left[m-\gamma q^{\prime \prime}\right]^{-1}\left[C \cdot A_{j}+S \cdot A_{j}\right]\right\}\left(m^{2}-q^{\prime 2}\right)^{-1}$.

A similar result is obtained for Fig. 12b. In this way the scalar propagator, the cubic scalar vertex $C$ and the magnetic-moment vertex $S$ emerge. The seagull scalar vertex is obtained by combining the first terms of Fig. 12a and $12 \mathrm{~b}$ to get $e^{2}\left\{\gamma A_{a}, \gamma A_{j}\right\}=2 e^{2} g^{\mu \nu} A_{a \mu} A_{j \nu} \rightarrow$ $Q^{\mu \nu} A_{\mu} A_{\nu}$.

At a vertex next to an incoming electron line with momentum $q^{\prime}$, the vertex factor $e \gamma A$ can be replaced by the factor $e \gamma A\left(m+\gamma q^{\prime}\right) / 2 m$, to enable (E1) to be used to derive (E2) and (E3). This accounts for the extra factor $(2 m)^{-1}$ per incoming electron. No factor is needed for an outgoing electron of momentum $q^{\prime \prime}$ because the second term on the lhs of (E1), when operating on the outgoing electron, is zero and can be added in freely. 


\section{References}

[1] F.A. Berends, R. Kleiss, P. De Causmaecker, R. Gastmans, W. Troost, and T.T. Wu, Phys. Lett. 103B (1981), 124; Nucl. Phys. B206 (1982), 61; ibid. 239 (1984), 382; ibid. 239 (1984), 395; ibid. 264 (1986), 243; ibid. 264 (1986), 265.

[2] P. De Causmaecker, R. Gastmans, W. Troost, and T.T. Wu, Phys. Lett. 105B (1981), 215; Nucl. Phys. B206 (1982), 53.

[3] Z. Xu, D.-H. Zhang, and L. Chang, Tsinghua University Preprints, Beijing, China, TUTP-84/4, TUTP-84/5, TUTP-84/6; Nucl. Phys. B291 (1987), 392.

[4] F.A. Berends and W.T. Giele, Nucl. Phys. B294 (1987), 700; ibid. 306 (1988), 759; ibid. 313 (1989), 595.

[5] F.A. Berends, W.T. Giele, and H. Kuijf, Phys. Lett. 211B (1988), 91; ibid. 232 (1989), 266; Nucl. Phys. B321 (1989), 39;ibid. 333 (1990), 120.

[6] J. Gunion and J. Kalinowski, Phys. Rev. D34 (1986), 2119.

[7] J. Gunion and Z. Kunszt, Phys. Lett. 159B (1985), 167; ibid. 161 (1985), 333; ibid. 176 (1986), 477.

[8] K. Hagiwara and D. Zeppenfeld, Nucl. Phys. B313 (1989), 560.

[9] R. Kleiss and H. Kuijf, Nucl. Phys. B312 (1989), 616.

[10] R. Kleiss and W.J. Stirling, Nucl. Phys. B262 (1985), 235.

[11] D. Kosower, Nucl. Phys. B315 (1989), 391; ibid. 335 (1990), 23.

[12] J.G. Körner and P. Sieben, Nucl. Phys. B363 (1991), 65.

[13] Z. Kunzt, Nucl. Phys. B271 (1986), 333.

[14] M. Mangano, Nucl. Phys. B309 (1988), 461.

[15] M. Mangano, S. Parke, and Z. Xu, Nucl. Phys. B298 (1988), 653.

[16] M. Mangano and S.J. Parke, Nucl. Phys. B299 (1988), 673; Phys. Rev. D39 (1989), 758; Phys. Rep. 200 (1991), 301.

[17] Z. Bern and D.K. Kosower, Nucl. Phys. B362 (1991), 289.

[18] S. Parke and T. Taylor, Phys. Lett. 157B (1985), 81; Nucl. Phys. B269 (1986), 410; Phys. Rev. Lett. B56 (1986), 2459; Phys. Rev. D35 (1987), 313.

[19] C. Dunn and T.-M. Yan, Nucl. Phys. B352 (1989), 402.

[20] G. Mahlon and T.-M. Yan, Phys. Rev. D47 (1993), 1776; G. Mahlon, T.-M. Yan, and C. Dunn, Phys. Rev. D47 (1993), 1120.

[21] G. Mahlon, Cornell preprint CLNS 92/1154; Cornell University Ph.D. thesis.

[22] Z. Bern and D.K. Kosower, Phys. Rev. Lett. B66 (1991), 1669; Nucl. Phys. B379 (1992), 451; preprint Fermilab-Conf-91/71-T.

[23] Z. Bern and D.C. Dunbar, Nucl. Phys. B379 (1992), 562.

[24] Z. Bern, L. Dixon, and D.A. Kosower, Phys. Rev. Lett. B70 (1993), 2677.

[25] M. Strassler, Nucl. Phys. B385 (1992), 145; SLAC preprint SLAC-PUB-5978.

[26] C.S. Lam, Nucl. Phys. B397 (1993), 143.

[27] C.S. Lam, Phys. Rev. D48 (1993), 873.

[28] J.D. Bjorken, Standford Ph.D. thesis (1958); J.D. Bjorken and S.D. Drell, 'Relativistic Quantum Fields' (McGraw-Hill, 1965).

[29] K. Symanzik, Progress of Theoretical Physics 20 (1958), 690.

[30] C.S. Lam and J.P. Lebrun, Nuovo Cimento 59A (1969), 397. 
[31] N. Nakanishi, 'Graph Theory and Feynman Integrals' (Gordon and Breach, 1971).

[32] P. Cvitanović and T. Kinoshita, Phys. Rev. D10 (1974), 3978; Phys. Rev. D10 (1974), 3991.

[33] C.S. Lam, Nuovo Cimento 59A (1969), 422.

[34] J. Paton and Chan Hong-Mo, Nucl. Phys. B10 (1969), 519.

[35] M. Veltman, Nucl. Phys. B319 (1989), 253.

[36] D. Kosower, Phys. Lett. 254B (1991), 439.

[37] L.F. Abbott, Nucl. Phys. B185 (1981), 189; L.F. Abott, M.T. Grisaru, and R.K. Schaefer, Nucl. Phys. B229 (1983), 372. 


\section{Figure Captions}

Fig. 1. A QCD diagram with internal momenta $q_{r}$ and Feynman (Schwinger) parameters $\alpha_{r}$. Each external fermion line is lablelled by (momentum, color, helicity).

Fig. 2. A QCD diagram with internal momenta $q_{r}$ and external momenta $p_{i}$. Each external line is labelled by (momentum, color).

Fig. 3. A QED or QCD diagram with internal momenta $q_{r}$ and external momenta $p_{i}$. The external line labels are (momentum, helicity).

Fig. 4. A QED or QCD diagram with internal momenta $q_{r}$ and external momenta $p_{i}$. The external line labels are (momentum, helicity).

Fig. 5. Modified diagram of Fig. 4 used to compute spinor paths and spin factors. $k_{i}$ are the reference momentum of the gluon whose actual momentum is $p_{i}$.

Fig. 6. Color-oriented QCD vertices in the background gauge used in color and spin paths. The quark, gluon, and ghost lines are respectively represented by a thick solid line, a thin solid line, and a dashed line. External gluon lines are indicated by a circled 'A'. Color is arranged in clockwise order, with color factors given by the trace of products of generators in that order for gluons and ghosts. For the quark-gluon vertex in diagram (t), the color factor is $T^{a}$. All momenta are assumed to be outgoing, with the spin factors written below the diagrams. The Lorentz index is that of the dislodged line, the presence of the coupling constant $g$ or $g^{2}$ is understood. Because of the unusual normalization (3.3), this coupling constant $g$ is $1 / \sqrt{2}$ of the usual one. For example, if the (momentum, Lorentz index, color index) labels of line 1 is $\left(q_{1}, \alpha, a\right)$, of line 2 is $\left(q_{2}, \beta, b\right)$, etc., the total vertex factor for Fig. $6(\mathrm{a})$ is $g \operatorname{Tr}\left(T^{a} T^{b} T^{c}\right)\left(q_{2}-q_{3}\right)_{\alpha}$, and for Fig. 6(e) is $+2 g^{2} \operatorname{Tr}\left(T^{a} T^{b} T^{c} T^{d}\right) g_{\alpha \gamma} g_{\beta \delta}$, and for Fig. 6(t) is $g T^{a} \gamma_{\alpha}$.

Fig. 7. A spinor path for Fig. 2, where $k_{i}$ is the reference momentum for an external gluon whose actual momentum is $p_{i}$. Each external line is labelled by (momentum, helicity).

Fig. 8. A cubic electromagnetic vertex for scalar QED. The solid and dashed lines are respectively the charged scalar and photon lines.

Fig. 9. A quartic (seagull) vertex for scalar QED. The solid and dashed lines are respectively the charged scalar and photon lines.

Fig.10. $m$-loop vector paths for a pure QCD diagram. Here $m=6$.

Fig.11. Some other possible vector paths for the same $m$-loop as Fig. 10.

Fig.12. A figure used to demonstrate eq. (A33).

Fig.13. A portion of a scalar QED diagram used to demonstrate local gauge invariance of an external photon line.

Fig.14. A portion of a scalar QED diagram used to demonstrate local gauge invariance of an internal photon line. 